\title{
Proteome-wide screening for mitogen-activated protein kinase docking motifs and interactors
}

Guangda Shi ${ }^{1}$, Jaylissa Torres Robles ${ }^{1,2}$, Claire Song ${ }^{1}$, Leonidas Salichos ${ }^{3}$, Hua Jane Lou ${ }^{1}$, Mark Gerstein ${ }^{3}$ and Benjamin E. Turk ${ }^{1^{*}}$

${ }^{1}$ Department of Pharmacology, Yale School of Medicine, New Haven, CT 06520, USA

${ }^{2}$ Department of Chemistry, Yale University, New Haven, CT 06520, USA

${ }^{3}$ Department of Molecular Biophysics and Biochemistry, Yale University, New Haven, CT 06520, USA

*Corresponding author. E-mail: ben.turk@yale.edu

One sentence summary: A proteome wide screen for kinase docking sequences reveals new interactors and motifs mediating selective targeting to JNK and p38 MAP kinases. 


\section{ABSTRACT}

Essential functions of mitogen-activated protein kinases (MAPKs) depend on their capacity to selectively phosphorylate a limited repertoire of substrates. MAPKs harbor a conserved groove located outside of the catalytic cleft that binds to short linear sequence motifs found in substrates and regulators. However, the weak and transient nature of these "docking" interactions poses a challenge to defining MAPK interactomes and associated sequence motifs. Here, we describe a yeast-based genetic screening pipeline to evaluate large collections of MAPK docking sequences in parallel. Using this platform we analyzed a combinatorial library based on the docking sequences from the MAPK kinases MKK6 and MKK7, defining features critical for binding to the stress-activated MAPKs JNK1 and p38 $\alpha$. We subsequently screened a library consisting of $\sim 12,000$ sequences from the human proteome, revealing a large number of MAPK-selective interactors, including many not conforming to previously defined docking motifs. Analysis of $\mathrm{p} 38 \alpha / \mathrm{JNK} 1$ exchange mutants identified specific docking groove residues mediating selective binding. Finally, we verified that docking sequences identified in the screen could function in substrate recruitment in vitro and in cultured cells. Collectively, these studies establish an approach for characterization of MAPK docking sequences and provide a resource for future investigation of signaling downstream of p38 and JNK MAP kinases. 


\section{INTRODUCTION}

Protein kinases function through phosphorylation of a limited number of effector substrates (1). Kinases achieve specificity in part through complementarity between the catalytic cleft and residues surrounding the site of phosphorylation. However, many kinases recognize minimal phosphorylation site motifs that are insufficient to confer protein-level selectivity, and closely related kinases with identical phosphorylation site specificity can nonetheless target distinct sets of substrates. A key example is the mitogen-activated protein kinases (MAPK) family, a group of Ser-Thr kinases conserved widely in eukaryotes (2). Canonical animal MAPKs, including the extracellular signal-regulated kinases (ERKs), c-Jun N-terminal kinases (JNKs) and p38 MAPKs (hereafter, p38), are positioned at the bottom of three-tiered kinase cascades activated in response to diverse cellular stimuli. The different MAPK subfamilies phosphorylate largely unique substrate repertoires to elicit distinct cellular responses, yet each targets a minimal Ser/Thr-Pro consensus sequence. MAPK specificity is at least in part driven by docking interactions, in which regions of the kinase outside of the catalytic cleft recruit substrates through binding sites distal from their sites of phosphorylation.

A major hub for MAPK docking interactions is the D-recruitment site (DRS), a conserved region of the catalytic domain that binds to substrates, scaffold proteins, MAPK kinases (MKKs) and MAPK phosphatases (MKPs) (3-5). Several highly conserved binding partners bind to the DRS via domains with intrinsic tertiary structure (6). However, the DRS more generally recognizes short linear motifs (SLiMs) termed D-sites found in unstructured regions of MAPK interactors (7-10). D-sites bind to MAPKs with moderate affinity $(\sim 100 \mathrm{nM}-30$ $\mu \mathrm{M})$, promoting transient kinase-substrate interactions and dynamic remodeling of signaling 
networks in response to stimuli (7). DRS engagement can also impact the conformation and dynamics of the MAPK catalytic domain, which may be important in promoting activation by MKKs and inactivation by MKPs (11-13).

The MAPK DRS forms a groove consisting of three adjacent hydrophobic pockets (designated the $\Phi_{\mathrm{L}}, \Phi_{\mathrm{A}}$ and $\Phi_{\mathrm{B}}$ pockets) and a proximal negatively charged "common docking” (CD) site $(7,8,14,15)$. D-site sequences include a cluster of basic residues complementary to the CD site, a variable linker, and two or three hydrophobic $(\phi)$ residues (in a $\phi_{\mathrm{L}}-\mathrm{X}-\mathrm{X}-\phi_{\mathrm{A}}-\mathrm{X}-\phi_{\mathrm{B}}$, $\phi_{\mathrm{L}}-\mathrm{X}-\phi_{\mathrm{A}}-\mathrm{X}-\phi_{\mathrm{B}}$, or $\phi_{\mathrm{A}}-\mathrm{X}-\phi_{\mathrm{B}}$ arrangement) that engage the corresponding $\Phi$ pockets $(10,15)$. Additional sequence features within the context of this general motif appear to confer selectivity for particular MAPK subfamilies $(7,9,16,17)$. Structural studies of MAPK-D-site complexes have revealed distinct binding modes associated with these subfamily-selective motifs, driven by arrangement of the hydrophobic residues as well as the sequence composition and bound conformation of the D-site linker sequence $(7,8,11,18-20)$.

Knowledge of these MAPK-selective D-site motifs has driven computational approaches to identify novel MAPK substrates by scanning protein databases for sequences similar to previously established interactors $(9,21)$. However, experimental approaches for the discovery of additional MAPK-selective sequence motifs are needed to better define MAPK interactomes and to understand how they assemble. Here, we describe a genetically encoded library screening platform to identify new MAPK-interacting D-sites that exploits the conservation of the core cascade from humans to budding yeast. We used this strategy to analyze a saturation mutagenesis library of MKK-derived D-sites interacting with the JNK1 and p38 $\alpha$ MAPKs and discovered previously unknown features of their corresponding motifs. We subsequently 
screened these MAPKs against a large library of human proteomic sequences, which allowed us to further refine the motifs and to identify new direct interaction partners. The screen differentiated between known p38 $\alpha$ and JNK1 interactors and identified an extensive number of binding partners that are selective for either kinase. The dataset therefore provides an unbiased resource to probe selective MAPK signaling pathways and will be an important resource for future investigation into MAPK biology.

\section{RESULTS}

\section{A yeast-based system for evaluation of MAPK docking sequences}

We exploited the evolutionary conservation of core MAPK cascades to enable screening complex D-site sequence libraries in budding yeast. Yeast require the p38/JNK ortholog Hog1 for adaptation to osmotic stress (22). Normal activation of the Hog1 cascade by hyperosmolarity induces transient cell cycle arrest, but constitutive signaling causes stable growth suppression (23) (Fig. 1A). Presumably because it is deregulated in yeast, we found that co-expression of mammalian MKK6 with $\mathrm{p} 38 \alpha$ was toxic to a strain lacking Hog 1 and its cognate MKK Pbs2 (Fig. S1). MKK6 harbors a D-site located upstream of the catalytic domain (Fig. 1B) that promotes its phosphorylation of p38 (24). Because growth suppression in the context of WT p38a, which partially autophosphorylates in yeast, occurred independently of the MKK6 D-site (Fig. S1), we examined a fully MKK-dependent p38 $\alpha$ mutant (L195A) (25). Growth impairment by $\mathrm{p} 38 \alpha^{\mathrm{L} 195 \mathrm{~A}}$ was less severe than for WT $\mathrm{p} 38 \alpha$, dependent on an intact MKK6 D-site, and partly alleviated by exchanging the MKK6 D-site with that of MKK7 $\left(\mathrm{MKK}^{\mathrm{D} 7}\right)$ (Fig. 1C). Furthermore, MKK6 ${ }^{\mathrm{D} 7}$, but not WT MKK6 or MKK6 ${ }^{\Delta \mathrm{D}}$, partly 
suppressed cell growth when co-expressed with the analogous JNK1 ${ }^{\text {L198A }}$ mutant (Fig. 1D). These results provide a system in which yeast growth is controlled by a D-site interaction with either the $\mathrm{p} 38 \alpha$ or JNK1 MAPK.

In order to evaluate D-site sequences for their MAPK binding capacity, we introduced a third component into this system by ectopically expressing a D-site peptide. We reasoned that engagement of the MAPK DRS by the D-site would rescue growth inhibition by blocking either MAPK-MKK or MAPK-substrate binding (Fig. 1E). We first engineered $h o g 1 \Delta p b s 2 \Delta$ strains harboring chromosomally integrated cassettes for constitutive expression of the MKK (WT MKK6 or MKK6 $\left.{ }^{\mathrm{D} 7}\right)$ and for inducible expression of the MAPK (p38 $\alpha^{\mathrm{L} 195 \mathrm{~A}}$ or JNK1 $\left.{ }^{\mathrm{L} 198 \mathrm{~A}}\right)$. We next introduced plasmids inducibly expressing D-site peptides fused to GST into these strains and assessed their growth under inducing or non-inducing conditions. Expression of p38 $\alpha-$ binding D-site peptides derived from MKK6 or MEF2A both substantially reversed growth impairment associated with co-expression of MKK6 and $\mathrm{p} 38 \alpha^{\mathrm{L} 195 \mathrm{~A}}$, while the MKK7 Dpeptide improved growth to a lesser extent (Fig. 1F). Likewise, D-site peptides from MKK7 and the JNK substrate NFAT4, but not from MKK6, rescued growth of the strain expressing $\mathrm{MKK}^{\mathrm{D} 7}$ and JNK1 ${ }^{\mathrm{L} 198 \mathrm{~A}}$ (Fig. 1G). These experiments establish a system in which expression of a MAPK-binding D-site is coupled to cell growth, providing the basis for screens to identify sequences engaging the MAPK DRS.

\section{Comprehensive mutagenesis of MAPK docking sites}

The importance of specific D-site residues in mediating selective interaction with MAPKs has been established by analysis of mutant proteins and synthetic peptides $(16,17,26,27)$, but 
low-throughput approaches can only analyze a limited number of variants. To more comprehensively analyze docking specificity using our yeast system, we designed a library of 715 unique sequences that included all possible single amino acid substitutions, as well as double Ala substitutions in all pairwise combinations, to D-sites from MKK6 and MKK7 (Fig. 2A, Data file S1). Oligonucleotides encoding these sequences were custom synthesized and cloned as a pool into the yeast GST fusion vector (Fig. 2B). Plasmid pools were introduced into MAPK/MKK-expressing yeast strains, and liquid cultures were expanded in derepressing (raffinose) media. A portion of the culture was reserved for sequencing, and the remainder was split and propagated under either inducing (raffinose + galactose) or non-inducing (glucose) conditions. At various times, cultures were sampled, plasmids extracted from cells, and the Dsite region PCR amplified with barcoding primers. PCR samples were pooled and analyzed by Illumina sequencing, providing the relative abundance of each component of the library at each time point. While no sequences changed substantially $(>10 \%)$ in relative abundance under noninducing conditions, library representation became skewed upon induction of either MAPK (Fig. 2C,D, Fig. S2, Data file S2). The change of representation of each variant over time was fit to an exponential function to provide a measure of its relative depletion or enrichment within the screen (Fig. 2C,D, Data file S3).

As anticipated, variants derived from cognate D-sites (MKK6 for $\mathrm{p} 38 \alpha$; MKK7 for JNK1) were generally enriched during selection in two separate experiments, while with few exceptions non-cognate D-sites were depleted (Fig 2D). Most substitutions to cognate D-sites resulted in slower growth rates, suggesting that the WT sequences are largely optimal, but for both $\mathrm{p} 38 \alpha$ and JNK1 some variants consistently conferred faster growth (Fig. 2E). For JNK1, 
Leu8 and Leu10, which engage the $\Phi_{\mathrm{L}}$ and $\Phi_{\mathrm{A}}$ pockets (13), appeared most critical for binding, while Leu12 that binds the $\Phi_{\mathrm{B}}$ pocket was more tolerant to substitution (Fig 2E,F,H). As anticipated, substitutions to the N-terminally positioned basic residues, Arg3 and Arg5, that bind to the CD region, were also deleterious. For JNK1 the largest improvement was observed by aromatic substitutions to Gln9. A Tyr residue located at the same position in the NFAT4 Dsite appears to provide intra- and intermolecular van der Waals contacts in the co-crystal structure with JNK1 (Fig. S3A) (7). For p38 $\alpha$, the most deleterious single (Fig. 2D) and double (Fig. 2F) amino acid substitutions were to Leu10 and Ile12, which engage the $\Phi_{\mathrm{A}}$ and $\Phi_{\mathrm{B}}$ pockets in the hydrophobic groove (Fig. 2I) (7, 28), where only hydrophobic residues were tolerated. As anticipated, substitutions to basic residues in the N-terminal cluster were also disfavored, and incorporation of additional Lys or Arg residues in this region appeared to promote binding. Surprisingly, mutating either Lys11 or Lys14 near the C-terminus, not previously reported as essential for interaction with $\mathrm{p} 38 \alpha$, also led to slower growth. Compared to JNK1, p38a was generally less tolerant of acidic residues, particularly in the C-terminal region (positions 11 - 14), likely related to a higher net negative charge in the DRS (see below). These observations suggest that $\mathrm{p} 38 \alpha$ D-site binding may be in part driven by bulk electrostatics, rather than position-specific ionic interactions. At two sites near the N-terminus, binding was apparently improved by substitution with hydrophobic residues that may access an additional hydrophobic pocket (termed the $\Phi_{\mathrm{U}}$ pocket) previously observed in some $\mathrm{p} 38$ and ERK D-site complexes (Fig. S3B) $(11,28)$. Only three residues within the MKK6 D-site sequence appeared suboptimal for binding, among them a Pro residue immediately downstream of the L-X-I motif. Overall, these positional scanning screens both confirmed elements of the 
MKK6 and MKK7 D-sites known to be critical for MAPK binding, and also identified additional features that favor or disfavor DRS interactions.

\section{Screening the human proteome for MAPK-interacting D-sites}

The screens described above allowed us to define key features of the MKK6 and MKK7 D-sites. However, it is established that different D-site peptides can assume distinct binding modes at the DRS, each associated with its own conformation and associated sequence motif $(7,9,11,13,19)$. To enable discovery of multiple MAPK-targeting motifs and to discover new p38 or JNK interactors, we designed a large library consisting of candidate D-site sequences derived from the human proteome following similar criteria to those used previously for in silico screens $(9,21)$ (Fig. 3A). We identified $~ 50,000$ occurrences of the general D-site motif (defined as $[\mathrm{RK}]-\mathrm{x}_{0-2}-[\mathrm{RK}]-\mathrm{x}_{3-5}-[\mathrm{ILV}]-\mathrm{x}-[\mathrm{FILMV}]$ ) in the human proteome. To increase the likelihood that sites would be accessible to bind MAPKs, we excluded sequences that fell within defined Pfam domains or were annotated to be extracellular or located within the ER or the Golgi apparatus. The remaining sequences were incorporated into a final library of 11,756 sequences from 5426 proteins, including most established JNK- and p38-interacting D-sites (Data file S1, Table S1).

Coding sequences for all components of the library were introduced as a pool into the yeast GST fusion vector and screened for interaction with $\mathrm{p} 38 \alpha$ and JNK1 as described above for the positional scanning libraries (Data file S4). Sequences were ranked by the average Z-score from three replicate screens (Data file S5). We observed established D-sites to be strongly enriched in screens for their respective MAPK (Fig 3B, data file S4). For JNK1, four of the 
five most highly ranked sequences (corresponding to JIP1, NFAT4, MKK4 and BMPR2) were previously reported docking sites. We defined "hits" as those sequences with an average Z score $\geq 2$ and $-\log _{10}(p$-value $) \geq 1$. By these criteria, all save one of the known JNK-interacting D-sites present in our library scored among the 133 hits in the JNK1 screen (Fig. 3B, Table S2). For p38 $\alpha$, we identified 71 hit sequences including four of seven known interacting Dsites (MKK4, MKK6, MEF2A and MEF2C). We note that the remaining D-sites (MKK3, PTPN5 and PTPN7) were also enriched in all screens but did not meet our hit threshold. We observed little overlap among hits for $\mathrm{p} 38 \alpha$ and JNK1 with only seven sequences on both lists (Fig. 3C), and no non-cognate D-sites scored as hits for either MAPK. The strong enrichment of previously known D-sites suggests that other hits are likely to include authentic MAPKinteracting sequences.

In order to validate results from our screens, we examined the capacity of individual enriched sequences to selectively bind p38 $\alpha$ and JNK1. To assess MAPK binding, we examined that ability of synthetic peptides to inhibit JNK1 and p38 $\alpha$ activity toward a fluorogenic substrate incorporating a requisite D-site (29). We chose 24 hit sequences that had not previously been established to bind the corresponding MAPKs. As a measure of relative binding affinity, we determined $\mathrm{IC}_{50}$ values for inhibition of both kinases from assays conducted at a range of competitor peptide concentrations (Fig. 4A, Table S3). We found that all sequences scoring as hits in the JNK1 screen indeed bound JNK1 with higher affinity than p38 $\alpha$ (Fig. 4A,B). While p38 $\alpha$ was favored by most of its hit sequences, one of them was nonselective, and two slightly favored JNK1. We noted a tendency for JNK1 to bind D-sites more tightly than $\mathrm{p} 38 \alpha$, with five having $\mathrm{IC}_{50}$ values below $2 \mu \mathrm{M}$ in comparison to only one for $\mathrm{p} 38 \alpha$ 
(Fig 4A). This phenomenon may reflect an intrinsic capacity for JNK1 to bind tightly to docking sites or a more stringent affinity cutoff for enrichment in the yeast-based screen. Overall, these experiments confirm that most sequences selected in our screen bind selectively to $\mathrm{p} 38 \alpha$ and JNK1 and do so with affinities comparable to established D-sites.

As D-sites are often found in MAPK substrates, we further examined the capacity for selected p38 and JNK-targeting sequences to function in substrate recruitment. For these experiments we used an N-terminal fragment of the JNK substrate NFAT4 that harbors a Dsite positioned upstream of established phosphorylation sites, including Ser165. We generated constructs in which the native NFAT4 D-site was either eliminated or substituted with hit sequences from the yeast screens. As anticipated, the WT fragment was robustly phosphorylated at Ser165 by JNK1 in vitro, and this was greatly reduced upon mutation of the docking site (Fig. 4C). Furthermore, substitution of four out of five JNK1 hit sequences tested fully restored Ser165 phosphorylation, while incorporation of $\mathrm{p} 38 \alpha$-targeting sites failed to do so. By contrast, $\mathrm{p} 38 \alpha$ poorly phosphorylated the WT fragment, while incorporating either of two of its hit sequences (L3MBTL3 and TRERF1) converted NFAT4 to a p38 $\alpha$ substrate. However, one other hit (SETD1B) failed to do so despite binding to p38 $\alpha$ with comparable affinity (Table S3). This observation suggests that in some cases, D-site binding is insufficient to promote phosphorylation of an associated protein. D-site engagement can impact MAPK conformational dynamics to promote kinase activity (30), and our results suggest that such effects may occur in a sequence-specific manner. We also note that one D-site that was exclusively a JNK1 hit (SYDE1) promoted phosphorylation by both MAPKs and was presumably a false negative in the $\mathrm{p} 38 \alpha$ screen. 


\section{D-site sequence motifs conferring MAPK selective binding}

Previous structural, biochemical, and computational analyses have identified multiple distinct sequence motifs selectively targeted by JNK and p38 MAPKs. Analysis of aligned hit sequences revealed that for both MAPKs, specific amino acids were significantly overrepresented at multiple positions in comparison to the full set of sequences in the library (Fig 5A). We expected that hit sequences for a given MAPK would conform to multiple distinct motifs that would not be evident from alignment of all hits. To deconvolute distinct motifs from the full dataset, we examined subsets of sequences in which a single overrepresented residue was fixed at one position (Fig. 5A). This analysis revealed two previously defined signatures within the JNK1 dataset: the "JIP class" motif (R-P-X-X-L-X-L) and the "NFAT4 class" motif (L-x-L-x-L/F) (9), with NFAT4 class sequences falling in one of two registers (with the first Leu residue occupying either position 8 or position 10 in the library). Among these core motifs, we observed specific residues to be enriched at intervening positions, for example hydrophilic residues downstream of the conserved Pro in the JIP class motif, and acidic/amidic residues intervening the $\phi_{\mathrm{A}}$ and $\phi_{\mathrm{B}}$ residues of both motifs. These two motif classes account for $82 \%$ of JNK1 hits, with the remaining sequences being enriched for a Leu residue in position 7 (producing a L-X-X-L-X-L motif) that likely occupies the $\Phi_{\mathrm{L}}$ pocket similar to the Pro residue in the JIP class motif. Hit sequences for p38 $\alpha$ were most strongly enriched for the previously defined "MEF2A class" motif (P/L-X-L-X-I/L-P) (9), with about 60\% harboring a Pro or aliphatic residue in position $8\left(\phi_{\mathrm{L}}\right)$ (Fig. 5B). Of the remaining sequences, about half had a hydrophobic residue in position 7 , and the other half were characterized by 
one or more basic residues upstream of the $\phi_{\mathrm{A}}$ (position 10) Leu residue. These residual sequences also lacked selectivity for an Ile residue at the $\phi_{\text {В }}$ position or a Pro residue at position 13, suggestive of a distinct binding mode. In keeping with the results from the positional scanning library above, proteomic sequences selected by p38 $\alpha$ were generally enriched for basic residues in positions proximal to the hydrophobic residues, a feature not generally considered as part of known interaction motifs. We note that there was no significant selection for Lys or Arg residues near the D-site N-terminus by either MAPK (Fig. 5A,B). However, because our library design included at least two basic residues in all sequences, they may still promote binding in a manner independently of their precise position.

We probed the importance of key elements of these motifs by substitution analysis of a p38 $\alpha$ hit peptide derived from KMT2D (RKTDRPALHLRIPP) in assays of kinase inhibition as described above (Fig. 5C). Consistent with our screens and previous reports that the identity of hydrophobic residues can drive MAPK specificity (16), we found that substitution of the $\phi_{\mathrm{B}}$ Ile residue with Phe led to a large decrease in p38 $\alpha$ binding, while modestly improving binding to JNK1. Combined substitution of the Ile12-Pro13 sequence with Leu-Ala also made the peptide more JNK1 selective, suggesting a switch to an NFAT4 motif class. Substitution of Arg12 with Glu had a larger impact on binding to $\mathrm{p} 38 \alpha$ in comparison to JNK, consistent with results from both the proteomic and positional scanning screens. Replacement of Asp4 with Arg improved binding to both MAPKs, confirming the importance of basic residues in that region. Collectively, these assays verify key sequence features selected by the two MAPKs in our screens.

Knowledge of a protein interaction motif can be used to discover new interacting proteins 
by searching databases for matching sequences (31). While our screening approach identifies MAPK interacting sequences present in the human proteome, authentic MAPK D-sites that do not conform strictly to the criteria used to build the library will be excluded. To identify D-sites not present within our screening library, we scanned the human proteome for sequences similar to our hits using the web application PSSMsearch (32). We performed separate searches using hit sequences corresponding to various $\mathrm{p} 38 \alpha$ and JNK1 targeting motifs (Table S4). As anticipated, these searches returned sequences from the yeast screens that were used to build the PSSM, as well as sequences that were absent from the library. We chose 8 peptides excluded from the original library and evaluated their affinities for $\mathrm{p} 38 \alpha$ and JNK1 in the competitive kinase assay (Table 1). With one exception, peptides bound to their predicted kinases with affinities comparable to those from the yeast screens $\left(\mathrm{IC}_{50}\right.$ range $0.3-5.6 \mu \mathrm{M}$ ). These results confirm our ability to computationally identify new docking sequences, providing additional validation for our experimentally determined motifs.

\section{Determinants of MAPK-selective D-site interactions}

We next considered features of the p38 and JNK DRS that might mediate selective targeting of distinct motifs. As noted above, p38 $\alpha$ was more generally selective for basic residues throughout the D-site sequence. Notably, the p38 $\alpha$ DRS is more negatively charged than that of JNK1, having nine acidic residues in comparison to four for JNK1. Unique acidic residues in p38 $\alpha$ cluster at two sites: the so-called $E D$ region proximal to the $\Phi_{\mathrm{L}}$ and $\Phi_{\mathrm{A}}$ pockets (4) and the CD region (Fig 5D). To examine the importance of these two regions to D-site specificity, we generated point mutants exchanging residues between p38 $\alpha$ and JNK1 and 
examined binding of peptides corresponding to MAPK-selective motifs by competitive kinase assay (Table 2). Mutating either the ED or CD regions of JNK1 to the corresponding residues in p38 $\alpha$ reduced binding of the JNK-selective NFAT4 and JIP1 peptides by an order of magnitude. While the JNK1 CD mutant had modestly increased affinity for the p38-selective MKK6 (MEF2A motif) and SETD1B (non-MEF2A motif) peptides, a larger effect was seen with the ED mutant, which had affinities similar to WT p38a for the two peptides. Conversely, the p38 $\alpha$ ED mutant decreased the affinity of both cognate peptides to levels comparable to those seen for JNK1, while the CD mutant was without significant effect. None of the p38a mutants conferred detectable binding to the JNK1 cognate NFAT4 and JIP1 peptides. These experiments substantiate a role for a specific cluster of residues in mediating selective binding of D-sites to p38 $\alpha$, either through specific side chain interactions or though bulk electrostatic effects.

\section{Discovery of docking-dependent MAPK substrates}

To explore how our D-site screens might contribute to our understanding of MAPK function, we first performed gene set enrichment analysis (33) to identify potential cellular processes invovling p38 and JNK (Table 3). Hits for p38 $\alpha$ were enriched for proteins involved in regulation of chromatin organization and gene transcription, with most $(65 \%)$ localizing to the nucleus. These observations are consistent with nuclear translocalization of activated p38 and for its established roles in transcriptional regulation (34), and suggest broader control of gene transcription than that has been previously appreciated. While the observed enrichment of JNK1 hits for proteins involved in the JNK MAPK cascade was expected due to the 
abundance of known interactors, this category also included four upstream regulators not previously known to interact with JNK (TNIK, NCOR1, MAGI3, CARD9) that could constitute points for feedback regulation. In addition, JNK1 interactors were significantly enriched for cytoskeleton-associated proteins and regulators of signaling by small GTPases.

Because D-sites can play a role in MAPK substrate recruitment, we reasoned that hits from our yeast screens include previously undescribed substrates. To verify that this was the case, we chose a pair of candidate substrates from our JNK1 screens, SYDE1 and SYDE2, that not previously known to interact with the kinase. SYDE1 and SYDE2 are GTPase activating proteins (GAPs) for RHO family small GTPases, with ascribed roles in embryonic development and neuronal function $(35,36)$. The JNK1-selected D-site sequences of SYDE1 and SYDE2 are found in an analogous position downstream of the GAP domain, though they belong to distinct motif classes (JIP and NFAT4-type, respectively). We found that JNK1 could phosphorylate purified SYDE1 and SYDE2 in vitro (Fig. 6A,B). Addition of a peptide corresponding to the NFAT4 D-site inhibited JNK1 activity toward both proteins, suggesting that phosphorylation was dependent on an interaction with the DRS. To determine whether they could be phosphorylated by JNK in cells, we transfected HEK293T cells with plasmids encoding either protein and treated cells with the protein synthesis inhibitor anisomycin to activate the JNK pathway in the presence or absence of the selective JNK inhibitor JNK-IN-8, and cell lysates were analyzed by Phos-tag SDS-PAGE and immunoblotting. We found that anisomycin induced phosphorylation of both proteins as judged by an electrophoretic mobility shift, which was reversed by treatment with the JNK inhibitor (Fig. 6C,D). Analysis of SYDE2 by liquid chromatography-tandem mass spectrometry (LC-MS/MS) revealed that a 
phosphorylation at a single residue (Ser1203) increased with anisomycin and decreased with JNK inhibitor treatment (Fig. 6E). This site is located 15 residues downstream of the SYDE2 D-site, consistent with prior observations that D-sites frequently direct MAPKs to phosphorylation sites proximal and downstream of the D-site (37). Overall, these observations verify that our ability to identify new MAPK substrates from our D-site screens.

\section{DISCUSSION}

Here we have described a genetic screening approach to the identification of kinase docking motifs and interacting sequences. Most prior screens for docking sequences have focused on protein phosphatases, which are generally described as lacking dephosphorylation site specificity and are hence dependent on non-catalytic interactions for substrate recruitment (38-41). However, there has been growing appreciation for the importance of non-catalytic SLiM-mediated interactions to kinase substrate targeting. For MAPKs, docking interactions can enforce selective targeting to individual subfamilies and can restrict the kinase to phosphorylate specific Ser or Thr residues in a given $\operatorname{substrate}(9,10,37)$. In other systems, docking is not absolutely required for phosphorylation in vivo, yet tuning the strength of such interactions can reportedly set phosphorylation rate. For example, SLiM-mediated recruitment to cyclin-dependent kinases through the cyclin subunit substrates controls the timing of phosphorylation within the cell division cycle $(42,43)$. In the case of the yeast LATS/NDR kinase Cbk1, an optimal docking sequence is not absolutely required for substrate phosphorylation yet confers robustness to perturbations that attenuate kinase activity (44).

Previous approaches to identify SLiMs mediating protein-protein or protein-enzyme 
interactions have used libraries of synthetic peptides or genetically-encoded phage display and cell surface display libraries $(40,45,46)$. Our method involving reconstitution of signaling pathways in yeast involves tunable pathway inhibition through a competitive interaction between kinases in the MAPK cascade or with downstream effectors. This approach may be advantageous in that interactions occur within a eukaryotic cell while maintaining sufficiently high throughput to enable extraction of binding motifs. It also enables discovery of interaction partners that might escape detection in MS-based proteomics experiments due to low abundance or restricted patterns of expression. The expression level of D-site fusion proteins in yeast sets a relatively stringent affinity threshold providing a low false positive rate, but we may consequently fail to identify low affinity interactions. For example, while our approach selected almost all known JNK interactors in our library, it failed to identify the established Dsite in the transcription factor ATF2. This is consistent with a recent report that regions of ATF2 outside of its SLiM make an additional contact with MAPKs to increase binding affinity (47). Likewise, the full p38 $\alpha$-interacting region of the phosphatase PTPN7/HePTP includes additional sequence flanking the canonical D-site $(48,49)$, and while enriched in our screen it fell below our hit threshold.

In this study we performed both a comprehensive mutagenesis screen of known docking sites as well as a screen of proteome-derived sequences. Screening positional scanning libraries has facilitated discovery of new kinase substrates conforming closely to the resulting motifs (50). Selection of proteomic libraries has an advantage in that it directly nominates candidate interaction partners and can discover high affinity sequences that might appear suboptimal. Furthermore, a sufficiently large set of interacting sequences will also provide key sequence 
features constituting the interaction motifs. Indeed, using our motifs we were able to identify additional high affinity JNK1 and p38 $\alpha$-binding sequences absent from our screening library. Our studies used a focused library built around a minimal consensus sequence shared by most known D-sites, excluding sites that bind in a reversed $\mathrm{N}-\mathrm{C}$ orientation such as those found in MAPKAPKs (7). In the future we plan to adapt our approach to screen more complex "disorderome" libraries (51) that are not limited by a particular mode of interaction and may facilitate discovery of additional interaction motifs.

Several previous studies have used computational approaches to scan proteomes for MAPK-interacting D-sites based on consensus motifs defined by alignment of known interactors and by integrating structural constraints based on crystallographic studies of MAPK-D-site complexes $(9,21)$. While we did observe substantial overlap in our datasets, the majority of our hit sequences were not previously predicted (see Table S2). For example, 36\% of JNK1 and $34 \%$ of our $\mathrm{p} 38 \alpha$ hits were also discovered in the structure-guided in silico screens conducted by Remenyi and co-workers (9), and 11\% of JNK1 hits had been identified by Bardwell and co-workers through database searches for sequences similar to known D-sites (21). We note that hits unique to our dataset were often dissimilar in sequence to those for which structural information is available. In particular over $90 \%$ of our $\mathrm{p} 38 \alpha$ hits lacking an $\phi_{\mathrm{L}}$ residue had not been previously predicted. These observations underscore the value of unbiased screens of proteome-derived libraries in the discovery of new interactors.

JNK and p38 MAPKs were originally identified as stress-activated kinases and are now known to have diverse roles in normal and disease physiology $(34,52,53)$. We found significant enrichment for specific processes associated with proteins harboring MAPK- 
interacting D-sites uncovered in our studies, suggestive of expanded roles for JNK and p38. For example, p38 is a well-established regulator of gene transcription, directly phosphorylating more than a dozen sequence-specific transcription factors (34). In addition to several previously unidentified transcription factor targets, approximately $25 \%$ of our hits were derived from chromatin-associated proteins, including multiple chromatin remodeling factors, components of lysine modification complexes, and methyllysine readers. These results suggest previously unappreciated mechanisms by which p38 may impact transcription. Furthermore, enrichment of GTPase regulators among JNK1 interactors is interesting in light of the capacity of RHO family GTPases to activate the JNK cascade $(54,55)$, and we verified that the RHO GAPs SYDE1 and SYDE2 are both cellular JNK substrates. JNK kinases may therefore have general roles in crosstalk or feedback regulation between GTPase signaling pathways. Overall, these studies provide a resource for further investigation into regulation of basic cellular processes by the p38 and JNK MAP kinases.

\section{MATERIALS AND METHODS}

\section{Plasmids}

The plasmid for constitutive expression of N-terminally HisMax epitope-tagged human MKK6 in yeast was generated by insertion of the full coding sequence (PCR amplified from pcDNA3HisMax-MKK6) (56) downstream of the $A C T 1$ promoter (PCR amplified from pGS62, a gift from Gavin Sherlock) in pRS416. MKK6 ${ }^{\Delta \mathrm{D}}$ and D-site substitution mutants on this background were generated by overlap extension PCR using mutagenic oligonucleotides to delete residues spanning Ser4 - Lys17 (SKGKKRNPGLKI) or replace them with D-sites from MKK7 
(PQRPRPTLQLPLAN), MEF2A (SRKPDLRVVIPPS) or NFAT4 (LERPSRDHLYLPLE).

To generate integrative expression vectors for WT MKK6 and MKK6 ${ }^{\mathrm{D} 7}$, coding sequences were first subcloned into pRS416-GPD downstream of the yeast TDH3 (GPD) promoter, and then the entire expression cassette was PCR amplified and cloned into the PacI and BglII sites of the plasmid HO-hisG-URA3-hisG-poly-HO (Addgene plasmid \#51661). The integrating inducible yeast expression vectors for N-terminally FLAG-tagged human JNK1 (isoform $\alpha_{1}$ ) and rat $\mathrm{p} 38 \alpha$ were generated by inserting the full-length coding sequences into pRS403-GAL1. The inducible yeast GST expression vector was generated by PCR amplifying the GST coding sequence from pGEX-4T1 and cloning into the SacI and SpeI sites of pRS416-GAL1. Coding sequences for individual D-sites together with the HisMax tag were then PCR amplified from the corresponding pRS416-MKK6 plasmid and inserted into the SpeI and ClaI sites downstream of GST.

Bacterial expression vectors for GST-JNK1 (pGEX4T1-3xFLAG-JNK1, Addgene \#47574), GST-p38a, His6-MKK4, His6-MKK6 ${ }^{\text {S207E/T211E }}$ (MKK6-EE), and constitutively active MEKK1 (MEKK-C) were previously reported (57, 58). Expression vectors for NFAT4 D-site variants were prepared by subcloning residues $3-407$ of WT NFAT4 from the corresponding mammalian expression vector (Addgene \#21664) into pGEX4T1, introducing ClaI and HindIII restriction sites flanking the D-site by site-directed mutagenesis, excising the native D-site coding sequence, and replacing it with synthetic oligonucleotide pairs harboring compatible ends.

The mammalian expression vector for human SYDE1 (Uniprot isoform 2, Q6ZW31-2) was generated by Gateway recombination from pDONR223-SYDE1 (human ORFeome 
collection) into the C-terminal 3xFLAG epitope tagged plasmid pV1900. The expression vector for N-terminally FLAG-tagged mouse SYDE2 was generated by PCR amplification of the coding sequence from pNICE HA-mSYD1B (Addgene \#59362) and insertion into pcDNA3-FLAG by Gibson assembly.

Point mutations in all plasmids were introduced by QuikChange site-directed mutagenesis.

\section{Design and generation of D-site libraries}

The positional scanning libraries consisted of all possible single amino acid substitutions and all double Ala mutations to the MKK6 (SKGKKRNPGLKIPK) and second MKK7 (PQRPRPTLQLPLAN) D-site. To design the proteomic library, we identified all sequences matching the regular expression $[\mathrm{RK}]-\mathrm{x}_{0-2}-[\mathrm{RK}]-\mathrm{x}_{3-5}-[\mathrm{ILV}]-\mathrm{x}-[\mathrm{FILMV}]$ within the human proteome. Sequences were extended at both termini to include two residues downstream of the motif and to bring the total length to 14 residues, and overlapping sequences were removed. Non-cytoplasmic sequences and those falling within annotated PFAM domains were excluded from the final library. Sequences were reverse translated in silico to yeast optimized codons, and silent mutations were introduced to remove restriction sites used for cloning. Common flanking sequences were added for separate PCR amplification of the positional scanning (5':

GCTTCAGGTGGACAACAATCACAA, 3': GAAGCTTCACTCTGTGTTGAAGTTCCGTCAG) and proteomic (5': GGTCGCGGATCTATGTCTCAG, 3’: GAAGCTTTTGAACAACCTCAGCAC) libraries. Core DNA and protein sequences are provided in Data file S1. Oligonucleotides were commercially synthesized as a pool (CustomArray), PCR-amplified, and restriction enzyme 
cloned into the NheI and HindIII sites downstream of the GST coding sequence of the pRS416GAL1-GST plasmid (see Fig. S4 for protein sequence). DH10ß cells (Invitrogen ElectroMAX) were transformed by electroporation with ligation products to produce at least 1000 transformants per library variant, and plasmid library DNA was prepared from pooled colonies. To ensure full representation of all components of the library, the variable region was PCRamplified and sequenced on an Illumina HiSeq 4000 instrument.

\section{Yeast growth assays}

Liquid cultures of the indicated strains transformed with the indicated plasmids were grown to mid-logarithmic phase in the appropriate selective media containing $2 \%$ raffinose. Aliquots of five-fold dilution series were spotted onto agar plates containing either $2 \%$ glucose or $2 \%$ raffinose $+1 \%$ galactose as indicated. Plates were incubated at $30^{\circ} \mathrm{C}$ for $48-96$ hours.

\section{Yeast-based screens}

The $S$. cerevisiae hog $1 \Delta$ pbs $2 \Delta$ strain was generated by PCR-based replacement of the entire $H O G 1$ open reading frame with the LEU2 marker in a $p b s 2 \triangle:$ KanMX strain from the yeast knockout collection (BY4741 strain background, Open Biosystems). The genotype was confirmed by diagnostic PCR of both deletion arms from genomic DNA. Strains used for screening were generated by subsequent integration of cassettes for galactose-inducible expression of $\mathrm{p} 38 \alpha^{\mathrm{L} 195 \mathrm{~A}}$ or JNK1 $1^{\mathrm{L} 198 \mathrm{~A}}$ (at the HIS3 locus) and constitutive GPD promoterdriven expression of His-tagged WT MKK6 or $\mathrm{MKK}^{\mathrm{D} 7}$ (at the $H O$ locus). Expression of MAPK and MKK6 alleles were confirmed by immunoblotting lysates from galactose-treated 
cells with antibodies to the FLAG and His6 tags, respectively.

Libraries of plasmids expressing D-site GST fusion proteins were introduced into yeast by LiOAc high-efficiency transformation (59) and selection on SC-Ura agar plates to produce at least 200 transformants per component. Transformed yeast were scraped from plates, pooled, diluted to an $\mathrm{OD}_{600}$ of 0.1 in SC-Ura liquid media with $2 \%$ glucose, and grown to saturation at $30^{\circ} \mathrm{C}$. Cells were diluted into SC-Ura with $2 \%$ raffinose and grown for 6 hours to derepress the GAL1 promoter. A starting time $\left(\mathrm{T}_{0}\right)$ sample (20 $\mathrm{OD}_{600}$ units) was reserved, and the remaining cells were split and diluted to an $\mathrm{OD}_{600}$ of 0.1 in either SC-Ura $+2 \%$ raffinose $+1 \%$ galactose (inducing conditions) or SC-Ura $+2 \%$ glucose (control conditions). Cultures were subjected to four growth and dilution cycles in which they were propagated until the induced culture reached an $\mathrm{OD}_{600}$ of $\sim 1.5$, a portion $\left(20 \mathrm{OD}_{600}\right.$ units) reserved, and remaining cells diluted in fresh pre-warmed media to an $\mathrm{OD}_{600}$ of 0.1 . Reserved cells were pelleted, washed once with sterile $\mathrm{dH}_{2} \mathrm{O}$, snap-frozen on dry ice/EtOH and stored at $-80^{\circ} \mathrm{C}$. Plasmids were extracted from each cell pellet and the D-site regions were PCR amplified, incorporating barcodes specific to each condition and time point and adaptors for sequencing. PCR products were agarose gelpurified, pooled, and subjected to Illumina sequencing (HiSeq 4000). The positional scanning library was screened twice, and the human proteomic library was screened three times against each MAPK.

Data were normalized to the total read counts for a given timepoint. Data for each sequence were fit to an exponential function in Microsoft Excel, where the inverse time constant $\lambda$ was calculated as the slope of the line of the $\log _{2}$ transformed fold-change in normalized read counts as a function of time, with the y-intercept set to zero. Z-scores for each sequence within an 
individual screen were calculated from $\left(\lambda_{\text {sequence }}-\lambda_{\text {mean }}\right) / \mathrm{SD}$. For the human proteomic screen, p-values were calculated by comparing the Z-scores for a given sequence against the Z-scores for all sequences in the library. The hit threshold $(Z \geq 2, p \leq 0.1)$ was chosen to maximize the number of true positives while excluding all true negatives.

\section{Database searches}

For PSSM searching, hit sequences were binned into four categories, accounting for sites to occur in a different register from our original definition. JIP class: all JNK1 hits containing a R-P-X-X- $\phi$ sequence starting at either position 4 or position 6 . NFAT4 class: JNK1 hits with an $\phi-\mathrm{X}-\phi-\mathrm{X}-\phi$ sequence starting at either position 8 or position 10 that were not included in the JIP class. MEF2 class: p38 $\alpha$ hit sequences with either a Leu or Pro residue at position 8 or a Pro residue at position 13. Other p38: p38 $\alpha$ hit sequences with a Lys or Arg residue at either position 8 or 9 , excluding sequences defined in the MEF2 motif. Sequences belonging to each motif class were entered into the program PSSMsearch (http://slim.icr.ac.uk/pssmsearch/) (32) and the resulting PSSM used to searched the human proteome with default settings (disorder cutoff $=0.4, p$-value cutoff $=0.001)$. Search results were ranked based on the PWM score, and top 200 sequences are shown in Table S4.

Gene set enrichment analysis for gene ontology categories associated with hit sequences was performed using the Broad Institute web interface (https://www.gsea-msigdb.org/).

\section{Protein Expression and Purification}

GST-tagged (JNK1, p38 $\alpha$, and NFAT4 ${ }^{3-407}$ variants) and His6-tagged (MKK6-EE and active 
MKK4 prepared by co-expression with MEKK-C) were expressed in BL21(DE3) E. coli and purified as described $(57,58)$. FLAG epitope-tagged SYDE1 and SYDE2 were expressed in and purified from polyethyleneimine-transfected HEK293T cells as previously described (60). The concentration and purity of protein preparations were assessed by SDS-PAGE and Coomassie Brilliant Blue R250 staining alongside BSA standards.

GST-p38 $\alpha$ and GST-JNK1 $(50 \mu \mathrm{M})$ were activated in vitro by incubation with $500 \mathrm{nM}$ His6-MKK6-EE or $50 \mathrm{nM}$ active His6-MKK4, respectively, in reaction buffer (50 mM Tris [pH 8.0], $100 \mathrm{mM} \mathrm{NaCl}, 10 \mathrm{mM} \mathrm{MgCl} 2,1 \mathrm{mM} \mathrm{DTT}, 0.012 \%$ Brij-35, $300 \mu \mathrm{M} \mathrm{ATP}$ ) at $30^{\circ} \mathrm{C}$ for 1.5 hours. Phosphorylation was confirmed by immunoblotting with anti-p38 pThr180/pTyr182 and JNK pThr183/pTyr185 antibodies as appropriate.

\section{Immunoblotting}

Samples for immunoblotting were fractionated by SDS-PAGE and transferred to polyvinylidene difluoride membranes. Membranes were blocked with 5\% non-fat dry milk in Tris buffered saline with $0.05 \%$ Tween-20 (TBS-T) at room temperature for 1 hour and probed with primary antibodies diluted according to the manufacturer's recommendation. The following primary antibodies used were obtained from Cell Signaling Technology: p38 pThr180/pTyr182 (\#9211), c-JUN (\#2315), c-JUN pSer63 (\#9261), GST (\#2624). Other antibodies used were: NFAT4 pSer165 (Sigma-Aldrich SAB4503947), FLAG M2 (SigmaAldrich F3165), vinculin (Sigma-Aldrich V9131), penta-His (Qiagen). Membranes were then incubated with fluorophore-conjugated secondary antibodies diluted 1:20,000 in TBS-T and 5\% BSA. The fluorescence signal was detected using an Odyssey CLx imaging system (LI-COR 
Biosciences) and quantified using Image Studio Lite software.

\section{MAPK D-site peptide inhibition assays}

D-site peptides were commercially synthesized (GenScript) incorporating a fixed Tyr-Ala sequence upstream of 14 residues corresponding to the yeast library sequence. Peptides were dissolved in DMSO to $10 \mathrm{mM}$ and stored at $-20^{\circ} \mathrm{C}$. MAPK assays were performed with a sulfonamido-oxine (SOX) containing substrate peptide (AssayQuant AQT0376). Kinase assays were performed in technical duplicate in black 384 well plates in reactions containing $50 \mathrm{mM}$ HEPES (pH 7.5), $10 \mathrm{mM} \mathrm{MgCl} 2,0.012 \%$ Brij-35, $1 \%$ glycerol, $0.2 \mathrm{mg} / \mathrm{ml} \mathrm{BSA}, 1 \mathrm{mM}$ ATP, $1.2 \mathrm{mM}$ DTT and $4 \mu \mathrm{M}$ SOX peptide substrate. Competitor D-site peptides were titrated in two-fold increments over a range from $31 \mathrm{nM}-64 \mu \mathrm{M}$. Reactions were initiated by adding activated $\mathrm{p} 38 \alpha$ or JNK1 to final concentrations of $3 \mathrm{nM}$ and $60 \mathrm{nM}$, respectively, and fluorescence (excitation $360 \mathrm{~nm}$, emission $485 \mathrm{~nm}$ ) was read every min over 1 hour in a Molecular Devices SpectraMax M5 plate reader. Initial velocities were calculated from the linear portions of the reaction progress curves. $\mathrm{IC}_{50}$ values and $95 \% \mathrm{CIs}$ were calculated by fitting data collected from three biological replicates to a sigmoidal dose-response curve using Prism 8.2.0 (GraphPad).

\section{Protein kinase assays}

GST-NFAT4 $4^{3-407}$ and its variants $(0.5 \mu \mathrm{M})$ were incubated with p38 $\alpha(14 \mathrm{nM})$ or JNK1 (7 $\mathrm{nM})$ in reaction buffer (50mM HEPES [pH 7.4], $100 \mathrm{mM} \mathrm{NaCl}, 10 \mathrm{mM} \mathrm{MgCl}, 0.012 \%$ Brij35, $1 \mathrm{mM}$ DTT, $1 \mathrm{mM} \mathrm{Na}_{3} \mathrm{VO}_{4}, 5 \mathrm{mM} \beta$-glycerophosphate, $100 \mu \mathrm{M} \mathrm{ATP}$ ) at $30^{\circ} \mathrm{C}$ for $20 \mathrm{~min}$. 
Reactions were quenched by adding SDS-PAGE loading buffer and analyzed by immunoblotting with antibodies to GST and NFAT4 pSer165.

Purified SYDE1 (100 nM) or SYDE2 (120 nM) was incubated with or without active JNK1 $(70 \mathrm{nM})$ in kinase reaction buffer containing $50 \mathrm{mM}$ HEPES (pH 7.4), $100 \mathrm{mM} \mathrm{NaCl}, 10 \mathrm{mM}$ $\mathrm{MgCl}_{2}, 0.012 \%$ Brij-35, $1 \mathrm{mM}$ DTT, $1 \mathrm{mM} \mathrm{Na} \mathrm{VO}_{4}, 1 \mathrm{mM} \beta$-glycerophosphate and $50 \mathrm{nM}$ staurosporine (to suppress background phosphorylation in the control reaction). Reactions were initiated by adding $\left[\gamma^{32}{ }^{32} \mathrm{P}\right] \mathrm{ATP}$ to a final concentration of $20 \mu \mathrm{M}$ at $0.1 \mu \mathrm{Ci} / \mu \mathrm{l}$. Reactions were incubated at $30^{\circ} \mathrm{C}$ for 20 min and then quenched with the addition of $5 \mu \mathrm{l}$ x SDS-PAGE loading buffer. Samples were separated by SDS-PAGE (10\% acrylamide) and gels were stained with Coomassie, destained, and exposed to a phosphor screen. Exposures were analyzed by phosphorimager and quantified using QuantityOne software (BioRad). Experiments were performed at least three times.

\section{Analysis of protein phosphorylation in cultured cells}

HEK293T cells were transiently transfected with FLAG-tagged SYDE1 and SYDE2 expression plasmids using polyethyleneimine. After $48 \mathrm{~h}$, cells were treated with either $5 \mu \mathrm{M}$ JNK-IN-8 (SelleckChem, S4901) or vehicle (0.1\% DMSO) for 1 hour followed by either 10 $\mu \mathrm{g} / \mathrm{mL}$ anisomycin or vehicle $\left(0.1 \%\right.$ DMSO) for an additional hour at $37^{\circ} \mathrm{C}$. Lysates were prepared as described, and a portion was subjected to either standard or Phos-Tag SDS-PAGE (61) followed by immunoblotting with antibodies to the FLAG epitope, total c-JUN, c-JUN pSer63, total JNK, phospho-JNK, and vinculin (all at 1:1000 dilution). Phos-Tag gels included 7.5\% acrylamide, $50 \mu \mathrm{M}$ Phos-Tag reagent (Nard Institute AAL-107), and $100 \mu \mathrm{M} \mathrm{MnCl}_{2}$. 
incubated in the dark 1 hour with fluorescently-labeled secondary antibodies diluted 1:20,000 in $5 \%$ non-fat milk in TBST. Membranes were washed with TBST on rocker at RT $3 \times 10$ minutes and imaged with Odyssey CLx (LI-COR Biosciences).

For MS analysis, FLAG-tagged proteins were isolated from $10 \mathrm{~cm}$ plates as described above and fractionated by SDS-PAGE. Gels were stained briefly with Coomassie Brilliant Blue and de-stained. Protein bands were excised and subjected to in-gel trypsin digestion and LCMS/MS analysis at the Yale Keck Biotechnology Resource Laboratory. 


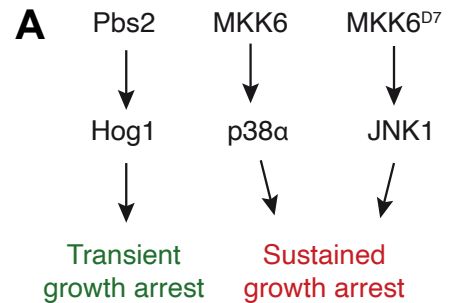

B

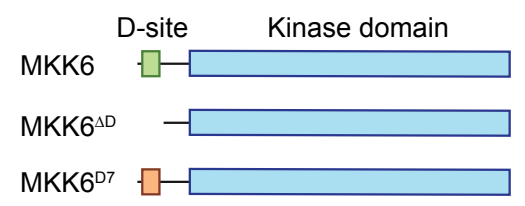

C Strain p38a MKK6 \begin{tabular}{c|cc} 
WT & - & - \\
$\operatorname{hog} 1 \Delta$ & + & - \\
pbs2 & + & WT \\
+ & $\Delta \mathrm{D}$ \\
+ & $\mathrm{D} 7$
\end{tabular}

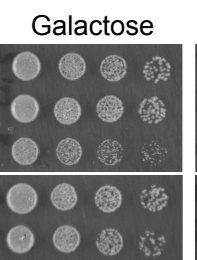

Glucose

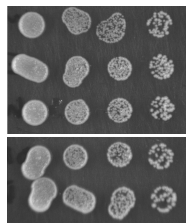

$\mathbf{F}$

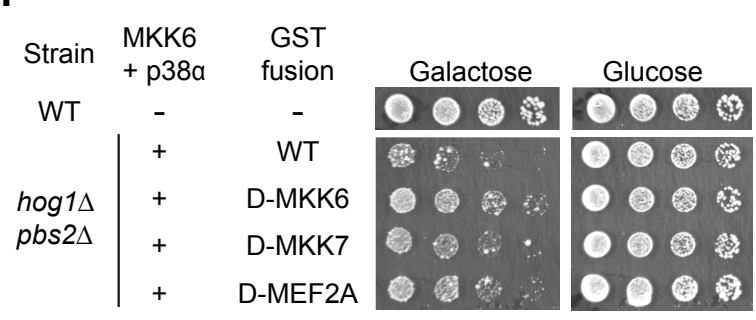

E

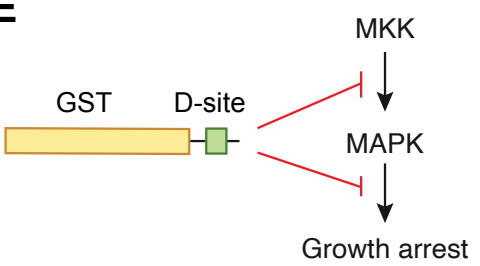

Figure 1. A system coupling MAPK D-site interactions to yeast cell growth. (A) Domain structure of MKK6 and D-site variants. $\mathrm{MKK}^{\mathrm{D} 7}$ replaces the native p38-selective D-site with that from MKK7, which binds only to JNK. (A) Scheme showing the impact of replacing yeast MAPK pathway components with their human homologs on cell growth. (C) Assay showing growth inhibition of a hog $1 \Delta$ pbs $2 \Delta$ strain upon co-expression of $\mathrm{p} 38 \alpha$ and the indicated MKK6 variants. Cells were grown in liquid culture, derepressed in raffinose media, and then spotted in 5-fold serial dilutions on solid media containing either galactose (to induce p38 expression) or glucose. (D) Growth assay of yeast co-expressing JNK1 and MKK6 variants conducted as in (C). (E) Scheme showing potential mechanisms for the growth rescue provided by expressing GST-D-site fusion proteins. (F) Effect of expressing cognate (MKK6, MEF2A) or non-cognate (MKK7) D-sites fused to GST on growth arrest mediated by p38 $\alpha$-MKK6 coexpression. Cells were grown and plated as in (C). (G) As (F) except with JNK1-MKK6 ${ }^{\text {D7 }}$ coexpression along with cognate (MKK7, NFAT4) or non-cognate (MKK6) D-sites. 

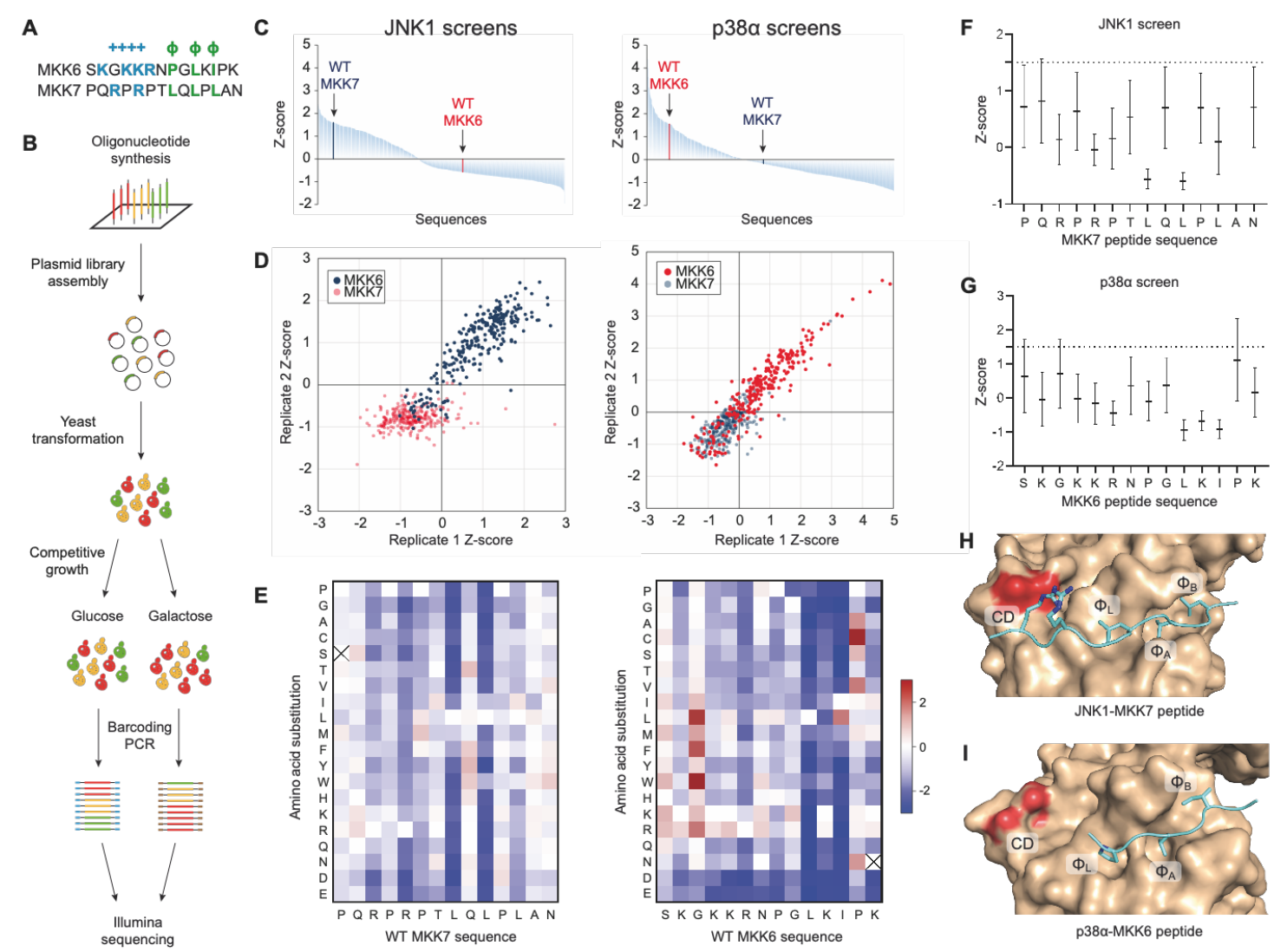

Figure 2. Combinatorial library screens. (A) A schematic showing the workflow of the screen. (B) Waterfall plots showing the average Z-score for the enrichment/depletion rate of each D-site sequence variant from two independent screens against JNK1 (left) or p38 $\alpha$ (right). WT MKK6 and MKK7 D-sites are highlighted. Read counts from Illumina sequencing runs are provided in Data file S1. (C) Scatter plot showing correlation of Z-scores between two replicate screens for JNK (left) and p38 (right). Blue, MKK6 variants; red, MKK7 variants. (D) Heatmaps showing the effect of each amino acid substitution to the MKK6 D-site in the p38a screen (left) and MKK7 D-site in the JNK screen (right). Values are normalized to the respective WT sequence (white), with red indicating enrichment and blue indicating depletion of the sequence from the population. Crossmarked boxes indicate sequences missing from the screening library. (E) Graph showing mean normalized enrichment scores of all double alanine substitutions to each position in the MKK7 D-site sequence in the JNK1 screen. The dotted line indicates value of the WT sequence. Error bars show SD for the 12 variants at that position. (F) Data for MKK6 double Ala variants in the p38a screen are plotted as in (E). (G) Crystal structures of JNK1 (tan) in complex with the MKK7 D-site (cyan) with the CD region and three hydrophobic pockets indicated (PDB entry 4UX9) (13). (H) As for panel (G), but showing p38 $\alpha$ bound to the MKK6 D-site (PDB entry 5ETF) (28). 
A Sequence motif
$[\mathrm{RK}]-\mathrm{x}\{0,2\}-[\mathrm{RK}]-\mathrm{x}\{3,5\}-[\mathrm{ILV}]-\mathrm{x}-[\mathrm{FILM}]$
$\sim 50,000$ human $14-$ mer sequences

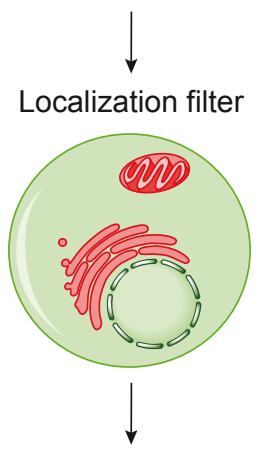

Structure filter

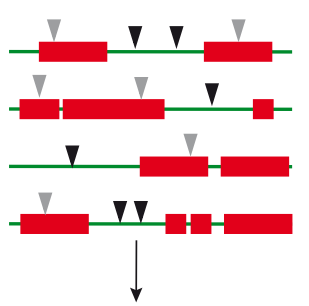

$\sim 12,000$ library sequences
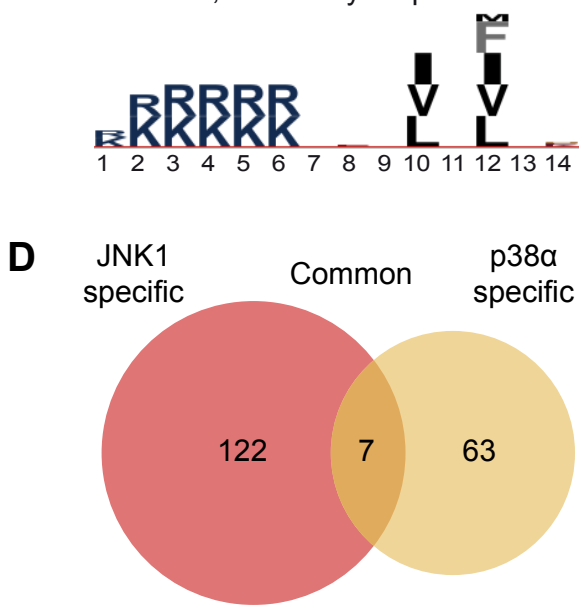

B
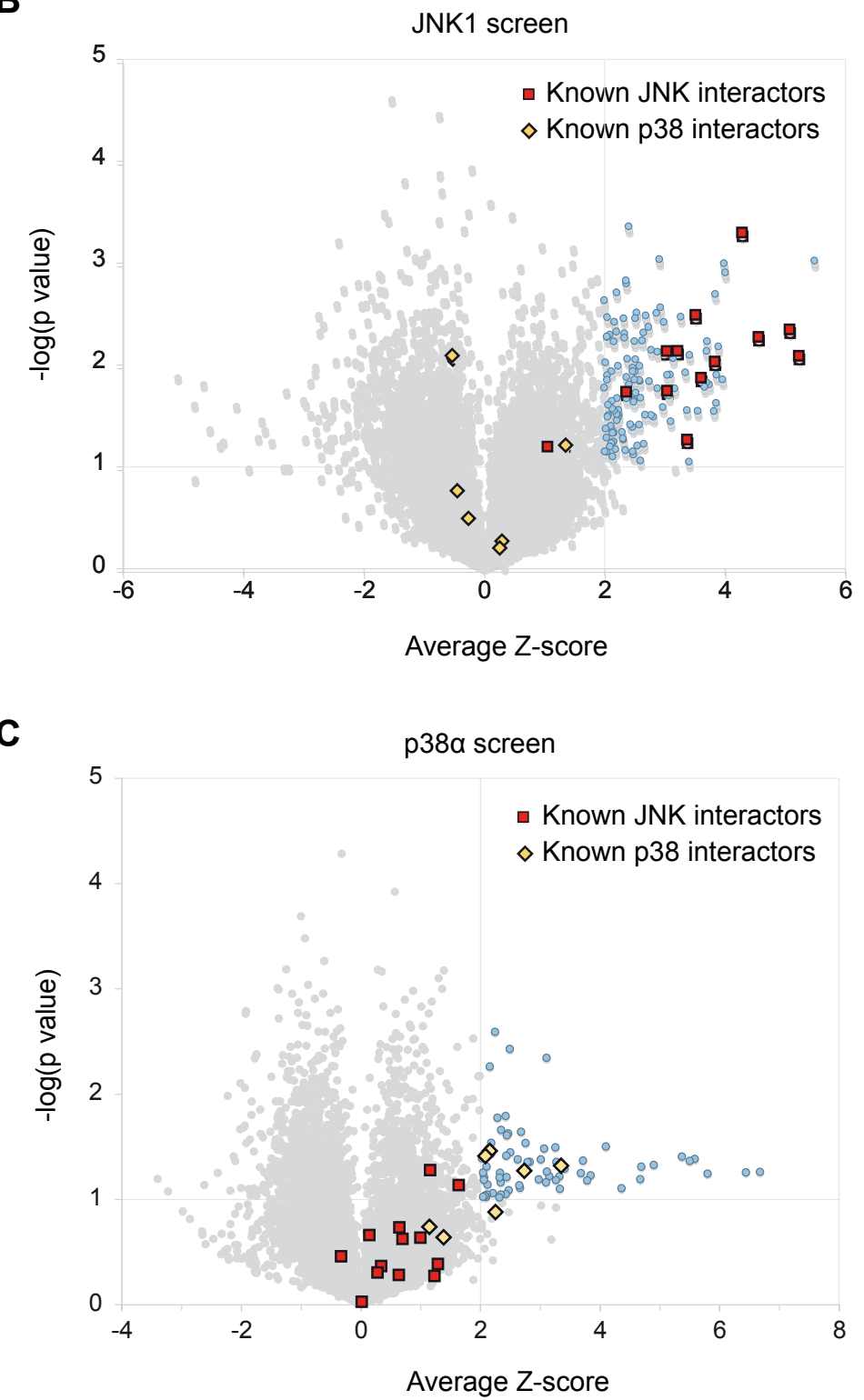

Figure 3. Proteomic library screens. (A) Schematic depicting selection of candidate D-site sequences from the human proteome. (B) and (C) Volcano plots for the JNK1 and p38 $\alpha$ screens, respectively, showing mean $\mathrm{Z}$-scores $(\mathrm{n}=3)$. The $p$-values (two-tailed heteroscedastic t-test) compare Z-scores for a given D-site with those of the entire population. Established functional JNK- and p38-interacting D-sites are indicated respectively as red squares and yellow diamonds. (D) Venn diagram showing overlap of hits $(Z \geq 2, p \leq 0.1)$ from the two screens. 
A

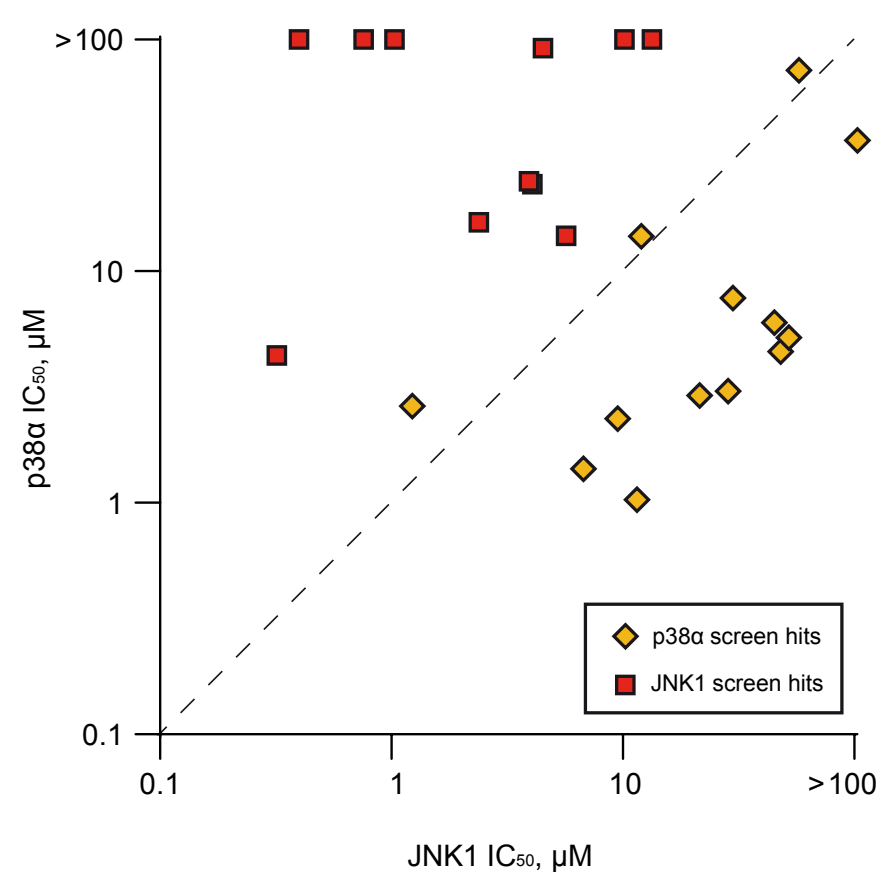

B

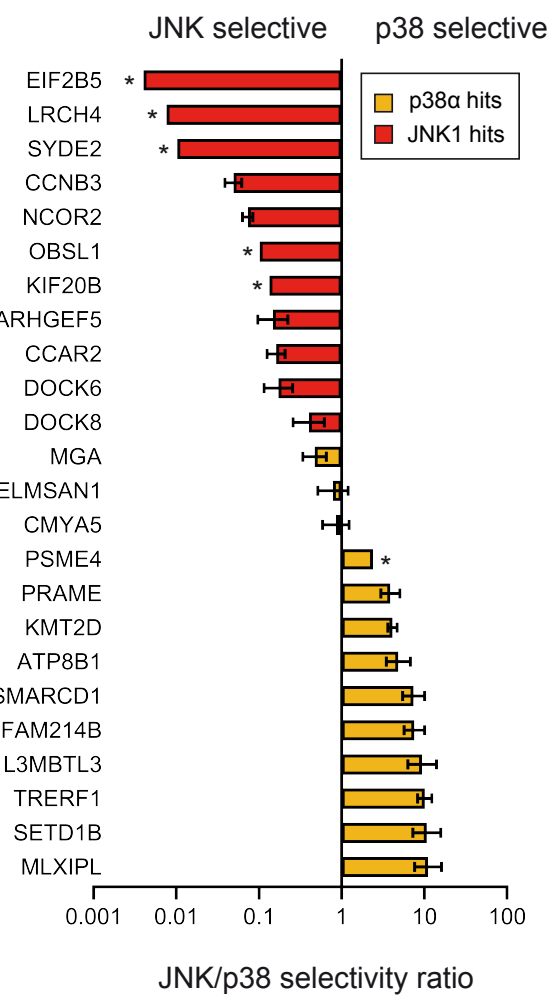

p38a activity

C

JNK1 activity

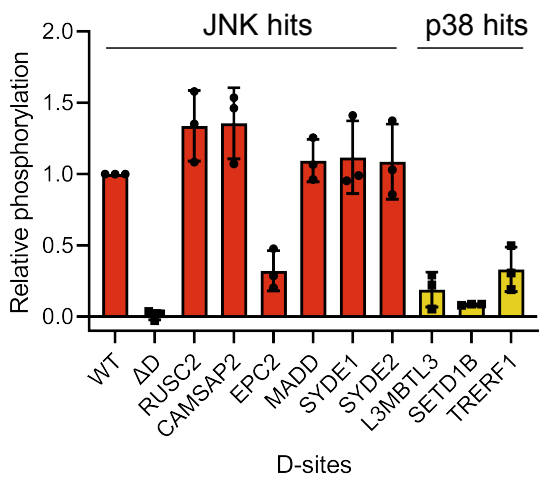

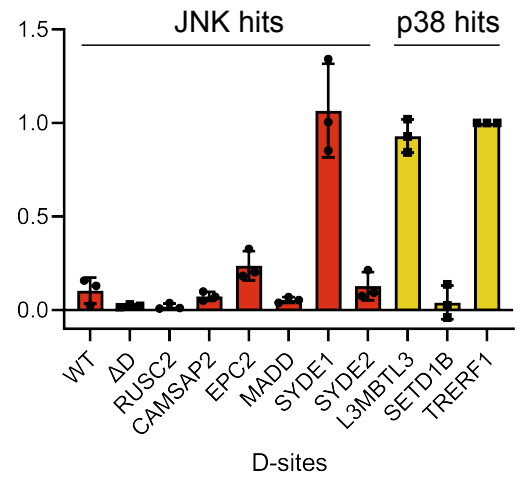

Figure 4. Hit validation. (A) Plot showing mean $\mathrm{IC}_{50}$ values $(\mathrm{n}=3)$ for inhibition of the indicated MAPK by each of 25 chemically synthesized hit peptides. $\mathrm{IC}_{50}$ values too high to be confidently determined for $\mathrm{p} 38 \alpha$ are indicated as being $>100 \mu \mathrm{M}$ and placed at the top edge. JNK1- and p38 $\alpha$-specific hits from the yeast screens are red squares and yellow diamonds, respectively. JNK1- and p38 $\alpha$-selective peptides fall respectively above and below the dotted line. (B) Graph shows ratios of JNK1 to $\mathrm{p} 38 \alpha \mathrm{IC}_{50}$ values for the indicated peptides, ordered from most JNK1-selective at top to most $\mathrm{p} 38 \alpha$-selective at bottom. For peptides binding weakly to $\mathrm{p} 38 \alpha$, the ratio was calculated using an IC50 value of $100 \mu \mathrm{M}$, and are indicated with an asterisk. Error bars show 95\% confidence intervals. (C) Graphs show relative level of phosphorylation by JNK1 (left) and p38 $\alpha$ (right) of chimeric NFAT4 constructs in which its D-site was replaced with those from the indicated hit proteins. Levels of phosphorylation were determined by immunoblotting with NFAT4 anti-phosphoSer165 antibody. Error bars indicate $\mathrm{SD}(\mathrm{n}=3)$. 
A
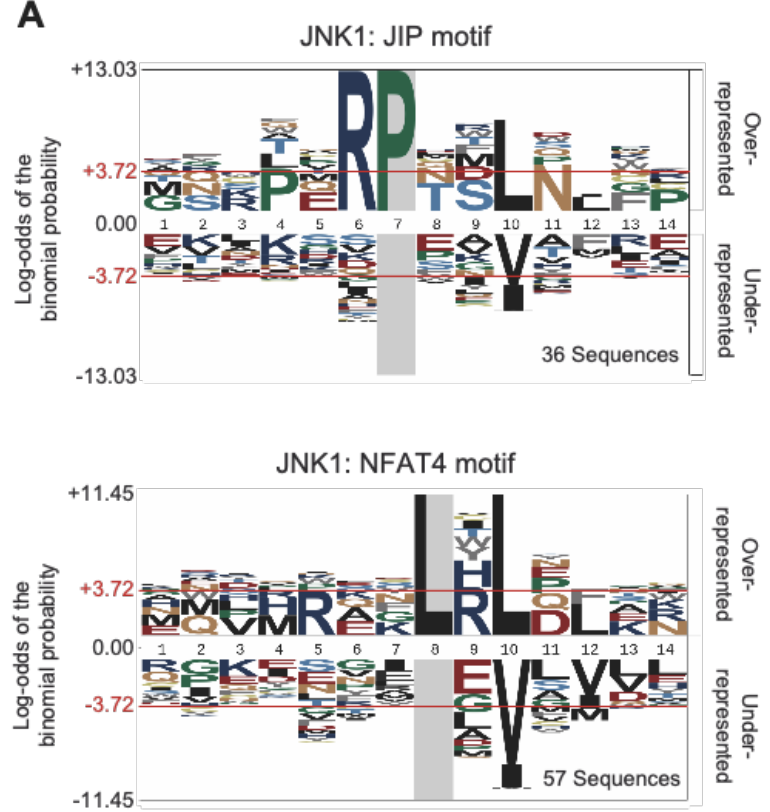

C

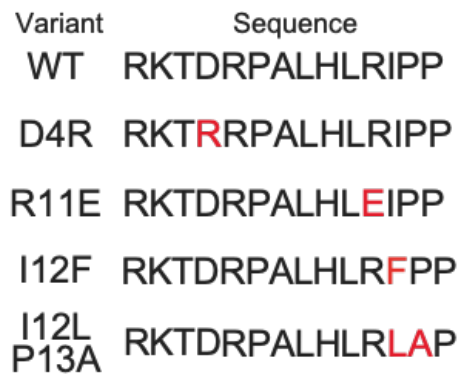

B
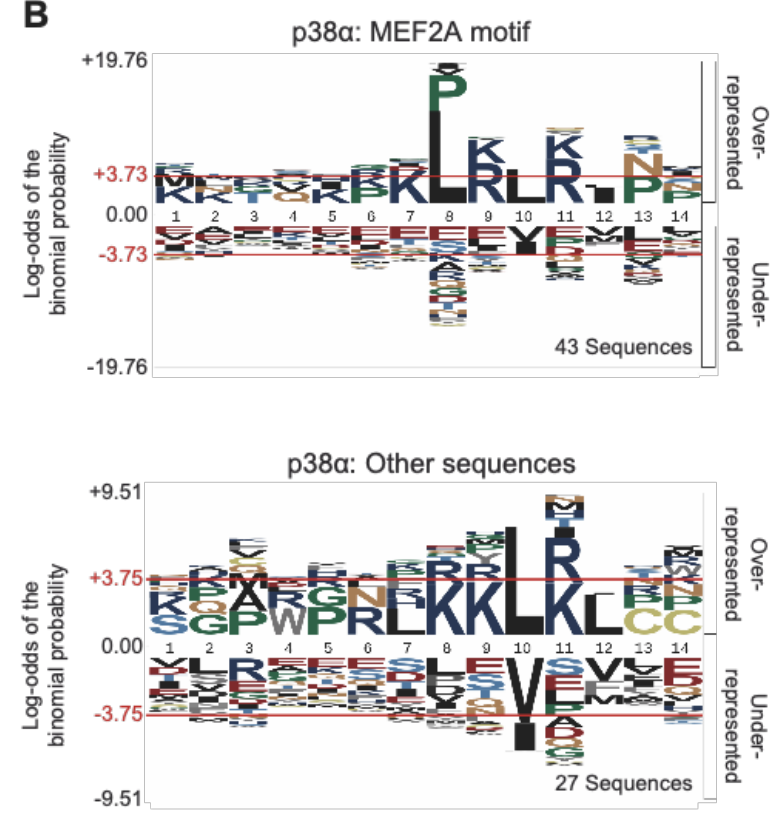

D

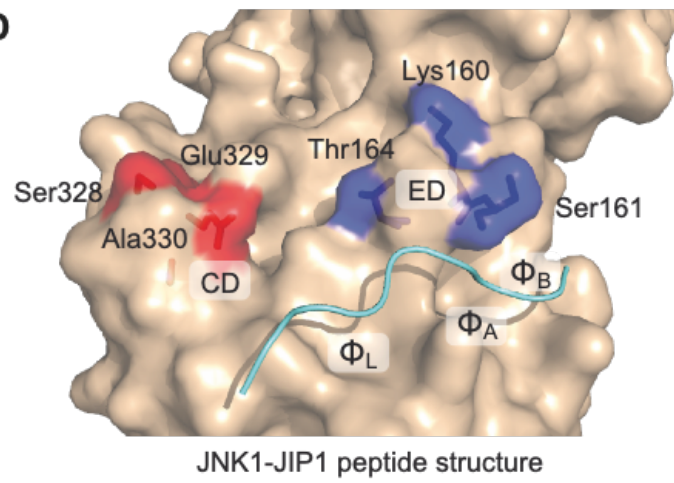

Figure 5. MAPK-interacting sequence motifs. (A) JNK1 hit sequences represented by pLogos(62). Top, subset of JNK1 hits having a Pro residue at position 7 (36 sequences) corresponding to the JIP class motif. Bottom, JNK1 hits with a Leu residue at position 8 (57 sequences) comprising the NFAT4 class motif. (B) Top, MEF2A motif class sequences including all p38a with either an Ile, Val, Leu, or Pro residue at position 8 or a Pro residue at position 13 (43 sequences). Bottom, all remaining p38a hits (27 sequences). (C) Peptide substitution analysis. Synthetic peptides corresponding to the $\mathrm{p} 38 \alpha$ hit KMT2D peptide and the indicated variants were evaluated for competitive inhibition of JNK1 or $\mathrm{p} 38 \alpha$ in vitro. Graph shows the average $(n=3)$ ratio of the $\mathrm{IC}_{50}$ value for the indicated point-substituted peptide to that of the WT peptide. Error bars show 95\% confidence intervals. (D) Potential D-site specificity-determining residues. Two patches of surface residues in the docking groove of JNK, the CD (red) and ED (blue) regions, are shown mapped on the crystal structure of JNK1 in complex with the JIP1 peptide (PDB code 1UKI) (18). 
A

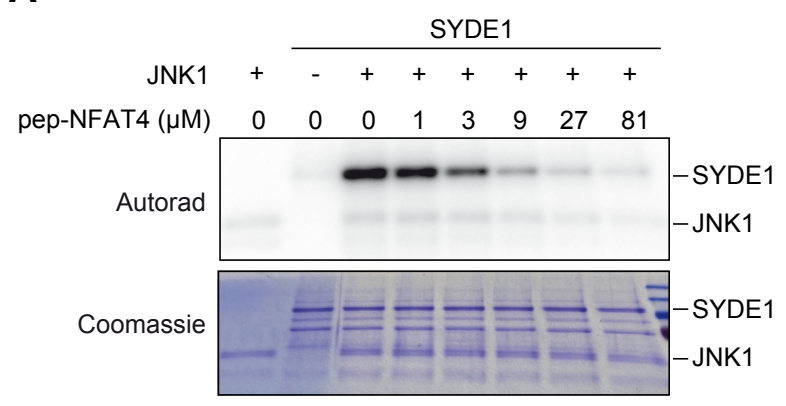

B

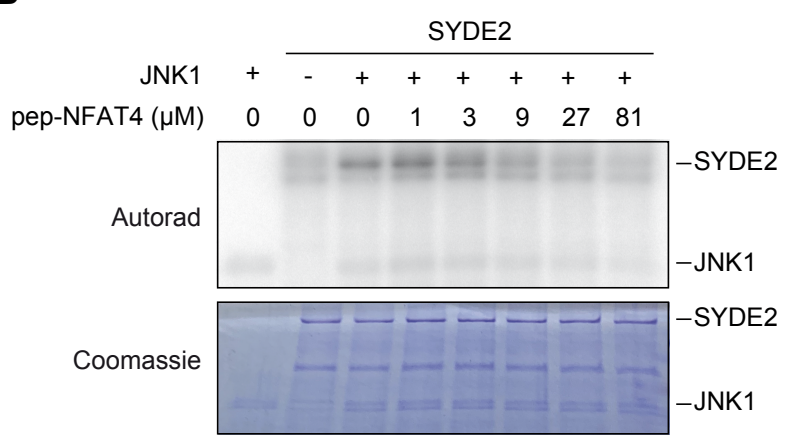

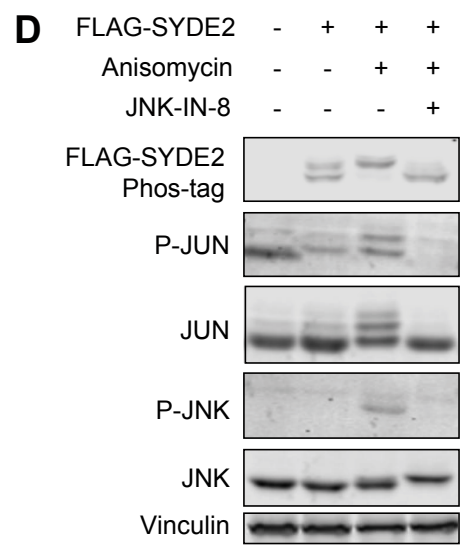

E

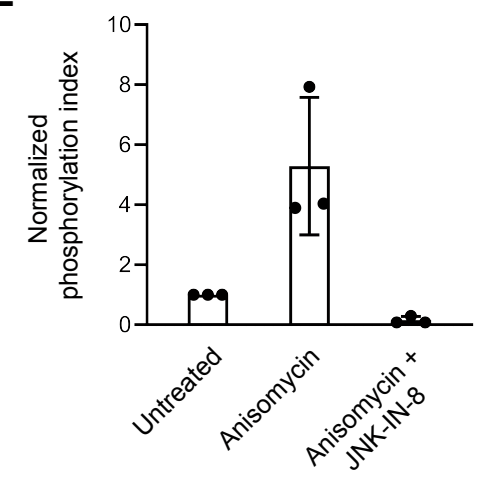

Figure 6. JNK substrate discovery. (A) In vitro radiolabel kinase assay showing JNK1 phosphorylation of full length SYDE1. Reactions were performed with increasing concentrations of the NFAT4 D-site competitor peptide to selectively block docking interactions. Representative images from three separate experiments are shown. (B) Kinase assay showing JNK1 phosphorylation of SYDE2, performed as in (A). (C) HEK293T cells transfected with a plasmid expressing FLAG-SYDE1 were treated with anisomycin following preincubation with or without the covalent JNK inhibitor JNK-IN-8. Lysates were subjected to either Phos-tag (top image) or standard (all others) SDS-PAGE, and immunoblotted with the indicated antibodies. (D) As in (C), except cells were transfected with a FLAG-SYDE2 expression vector. (E) MS analysis of SYDE2 phosphorylation. HEK293T cells transiently expressing FLAG-SYDE2 were treated as indicated. SYDE2 was immunoaffinity purified, and tryptic peptides were analyzed by LC-MS/MS. Graph shows the level of the Ser1082phosphorylated peptide normalized to the total abundance of the corresponding peptide. 


\section{TABLES}

Table 1. Inhibitory potency of peptide sequences identified by PSSMsearch.

\begin{tabular}{|c|c|c|c|c|c|}
\hline Protein & Sequence & $\begin{array}{l}\text { JNK1 } \\
\text { search } \\
\text { rank }\end{array}$ & $\begin{array}{l}\text { p38a } \\
\text { search } \\
\text { rank }\end{array}$ & $\begin{array}{l}\text { JNK1 IC }_{50}, \mu \mathrm{M} \\
(95 \% \text { CI })\end{array}$ & $\begin{array}{l}\text { p38 } \alpha \mathrm{IC}_{50}, \mu \mathrm{M} \\
(95 \% \mathrm{CI})\end{array}$ \\
\hline AFAP1L2 & KQVRKKEHKLKITP & - & 4 & $>100$ & $40(34-47)$ \\
\hline RASGRF1 & SPSRRRKLSLNIPI & 74 & 2 & $1.1(0.89-1.3)$ & $2.4(2.0-2.9)$ \\
\hline WDR3 & KRKRKKREKLILTL & 25 & 7 & $0.90(0.81-1.0)$ & $0.30(0.24-0.36)$ \\
\hline TTLL13P & RRRKRRSLAINLTN & 10 & 48 & $1.8(1.7-2.0)$ & $0.76(0.64-0.90)$ \\
\hline MXD4 & EKHRRAKLRLYLEQ & 1 & 34 & $1.1(0.95-1.3)$ & $5.2(3.5-7.8)$ \\
\hline MYH14 & GEQRRRRLELQLQE & 8 & - & $5.6(4.6-6.9)$ & $35(26-49)$ \\
\hline CDX1 & YPGPARPASLGLGP & 36 & - & $2.7(2.4-3.0)$ & $>100$ \\
\hline
\end{tabular}

Table 2. Impact of $\mathbf{p 3 8} \alpha / \mathrm{JNK} 1$ exchange mutagenesis on peptide binding affinities. JNK1

ED mutant, K160N/S161E/T164E; JNK1 CD mutant, S328D/E329D/A330E; p38a ED mutant,

N159K/E160S/E163T; p38 $\alpha$ CD mutant, D315S/D316E/E317A.

\begin{tabular}{|c|c|c|c|c|c|c|c|}
\hline \multirow[b]{2}{*}{ Peptide } & \multirow[b]{2}{*}{ Sequence } & \multicolumn{3}{|c|}{ JNK1 IC $_{50}, \mu \mathrm{M}(95 \%$ CI $)$} & \multicolumn{3}{|c|}{$\mathrm{p} 38 \alpha \mathrm{IC}_{50}, \mu \mathrm{M}(95 \% \mathrm{CI})$} \\
\hline & & WT & ED mutant & CD mutant & WT & ED mutant & CD mutant \\
\hline NFAT4 & ERPSRDHLYLPLEP & $0.89(0.76-1.0)$ & $9.5(8.6-10)$ & $\begin{array}{lll}6.3 & (5.0 \quad- \\
7.9) & & \\
\end{array}$ & $>100$ & $>100$ & $>100$ \\
\hline JIP3 & GRRKERPTSLNVFP & $\begin{array}{lll}0.36 & (0.31 & - \\
0.43) & & \\
\end{array}$ & $\begin{array}{lll}2.5 & (2.2 \quad- \\
2.8) & & \\
\end{array}$ & 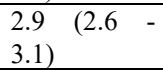 & $>100$ & $>100$ & $>100$ \\
\hline MKK6 & SKGKKRNPGLKIPK & $95(78-120)$ & $24(21-27)$ & $75(53-110)$ & $16(14-19)$ & $65(57-76)$ & $16(14-18)$ \\
\hline SETD1B & NQLKFRKKKLKFCK & $28(25-33)$ & $\begin{array}{lll}2.0 & (1.6 \quad- \\
2.4) & & \\
\end{array}$ & $14(7.4-28)$ & $\begin{array}{lll}7.8 & (6.3 \quad- \\
9.7) & & \\
\end{array}$ & $30(25-37)$ & $\begin{array}{lll}8.0 & (6.4 & - \\
10) & & \\
\end{array}$ \\
\hline
\end{tabular}

Table 3. Gene set enrichment analysis of $\mathbf{p 3 8} \alpha$ and JNK1 hits. FDR, false discovery rate.

\begin{tabular}{|l|c|l|c|}
\hline \multicolumn{2}{|c|}{ JNK1 screen } & \multicolumn{2}{c|}{ p38a screen } \\
\hline GO category & FDR q-value & GO category & FDR q-value \\
\hline $\begin{array}{l}\text { Regulation of Intracellular } \\
\text { Signal Transduction }\end{array}$ & $1.44 \mathrm{E}-10$ & $\begin{array}{l}\text { Chromatin } \\
\text { Organization }\end{array}$ & $9.80 \mathrm{E}-09$ \\
\hline $\begin{array}{l}\text { Nucleoside Triphosphatase } \\
\text { Regulator Activity }\end{array}$ & $5.82 \mathrm{E}-10$ & $\begin{array}{l}\text { Chromosome } \\
\text { Organization }\end{array}$ & $2.73 \mathrm{E}-08$ \\
\hline Cytoskeleton Organization & $1.65 \mathrm{E}-09$ & Catalytic Complex & $1.19 \mathrm{E}-07$ \\
\hline $\begin{array}{l}\text { Small GTPase Mediated } \\
\text { Signal Transduction }\end{array}$ & $2.85 \mathrm{E}-09$ & $\begin{array}{l}\text { Positive Regulation of } \\
\text { Biosynthetic Process }\end{array}$ & $3.54 \mathrm{E}-07$ \\
\hline $\begin{array}{l}\text { Guanyl Nucleotide Exchange } \\
\text { Factor Activity }\end{array}$ & $7.90 \mathrm{E}-09$ & $\begin{array}{l}\text { Positive Regulation of } \\
\text { Nucleobase } \\
\text { Containing Compound } \\
\text { Metabolic Process }\end{array}$ & $8.98 \mathrm{E}-07$ \\
\hline $\begin{array}{l}\text { Positive Regulation of } \\
\text { Catalytic Activity }\end{array}$ & $8.41 \mathrm{E}-09$ & $\begin{array}{l}\text { Nuclear Protein } \\
\text { Containing Complex }\end{array}$ & $8.98 \mathrm{E}-07$ \\
\hline
\end{tabular}




\section{SUPPLEMENTARY MATERIALS}

Figure S1. Growth suppression from co-expression of MKK6 and p38 $\alpha$ in yeast.

Figure S2. Yeast competitive growth screens.

Figure S3. Structures of p38 $\alpha$ and JNK1 in complex with D-site peptides.

Figure S4. Amino acid sequences of GST fusion libraries.

Data file S1. Amino acid and encoding nucleotide sequences for all components of the positional scanning and proteomic libraries.

Data file S2. Raw sequencing data for combinatorial library screens.

Data file S3. Z-scores for combinatorial library screens.

Data file S4. Raw sequencing data for proteomic library screens.

Data file S5. Z scores for proteomic library screens.

Table S1. List of previously reported functional D-sites present in the proteomic library.

Table S2. Hits from proteomic library screens.

Table S3. IC 50 values for D-site peptide inhibition of MAPK activity.

Table S4. PSSMsearch results.

\section{ACKNOWLEDGMENTS}

We thank Elias Lolis, Anton Bennett, Joel Sexton, and Erik Schaefer for advice and suggestions regarding this work, and we thank Titus Boggon for advice and feedback on the manuscript. We thank Karl Barber for assistance with oligonucleotide library design, and TuKiet Lam for LC-MS/MS analysis. We thank Ana Thevenin and Gavin Sherlock for providing plasmids, and the following investigators from whom plasmids were obtained through Addgene: Anjana Rao, Peter Scheiffele, David Stillman and Kevin Janes. This work 
was supported by National Institutes of Health grant R01 GM135331 to B.E.T. Additional support was provided by the China Scholarship Council to G.S., a National Science Foundation Graduate Research Fellowship to J.T.R. and NIH T32 GM007324 to C.S.

\section{REFERENCES}

1. C. J. Miller, B. E. Turk, Homing in: Mechanisms of Substrate Targeting by Protein Kinases. Trends Biochem Sci 43, 380-394 (2018).

2. M. Raman, W. Chen, M. H. Cobb, Differential regulation and properties of MAPKs. Oncogene 26, 3100-3112 (2007).

3. W. Peti, R. Page, Molecular basis of MAP kinase regulation. Protein Sci 22, 1698-1710 (2013).

4. T. Tanoue, R. Maeda, M. Adachi, E. Nishida, Identification of a docking groove on ERK and p38 MAP kinases that regulates the specificity of docking interactions. EMBO $J$ 20, 466-479 (2001).

5. E. J. Goldsmith, R. Akella, X. Min, T. Zhou, J. M. Humphreys, Substrate and docking interactions in serine/threonine protein kinases. Chem Rev 107, 5065-5081 (2007).

6. Y. Y. Zhang, J. W. Wu, Z. X. Wang, A distinct interaction mode revealed by the crystal structure of the kinase p38 $\alpha$ with the MAPK binding domain of the phosphatase MKP5. Sci Signal 4, ra88 (2011).

7. A. Garai, A. Zeke, G. Gogl, I. Toro, F. Fordos, H. Blankenburg, T. Barkai, J. Varga, A. Alexa, D. Emig, M. Albrecht, A. Remenyi, Specificity of linear motifs that bind to a common mitogen-activated protein kinase docking groove. Sci Signa. 5, ra74 (2012).

8. C. I. Chang, B. E. Xu, R. Akella, M. H. Cobb, E. J. Goldsmith, Crystal structures of MAP kinase p38 complexed to the docking sites on its nuclear substrate MEF2A and activator MKK3b. Mol Cell 9, 1241-1249 (2002).

9. A. Zeke, T. Bastys, A. Alexa, A. Garai, B. Meszaros, K. Kirsch, Z. Dosztanyi, O. V. Kalinina, A. Remenyi, Systematic discovery of linear binding motifs targeting an ancient protein interaction surface on MAP kinases. Mol Syst Biol 11, 837 (2015).

10. A. D. Sharrocks, S. H. Yang, A. Galanis, Docking domains and substrate-specificity determination for MAP kinases. Trends Biochem Sci 25, 448-453 (2000).

11. T. Zhou, L. Sun, J. Humphreys, E. J. Goldsmith, Docking interactions induce exposure of activation loop in the MAP kinase ERK2. Structure 14, 1011-1019 (2006). 
12. G. S. Kumar, H. Zettl, R. Page, W. Peti, Structural basis for the regulation of the mitogen-activated protein (MAP) kinase $\mathrm{p} 38 \alpha$ by the dual specificity phosphatase 16 MAP kinase binding domain in solution. J Biol Chem 288, 28347-28356 (2013).

13. J. Kragelj, A. Palencia, M. H. Nanao, D. Maurin, G. Bouvignies, M. Blackledge, M. R. Jensen, Structure and dynamics of the MKK7-JNK signaling complex. Proc Natl Acad Sci U S A 112, 3409-3414 (2015).

14. B. Xu, S. Stippec, F. L. Robinson, M. H. Cobb, Hydrophobic as well as charged residues in both MEK1 and ERK2 are important for their proper docking. J Biol Chem 276, 26509-26515 (2001).

15. T. Tanoue, M. Adachi, T. Moriguchi, E. Nishida, A conserved docking motif in MAP kinases common to substrates, activators and regulators. Nat Cell Biol 2, 110-116 (2000).

16. A. J. Bardwell, L. Bardwell, Two hydrophobic residues can determine the specificity of mitogen-activated protein kinase docking interactions. J Biol Chem 290, 26661-26674 (2015).

17. D. Barsyte-Lovejoy, A. Galanis, A. D. Sharrocks, Specificity determinants in MAPK signaling to transcription factors. J Biol Chem 277, 9896-9903 (2002).

18. Y. S. Heo, S. K. Kim, C. I. Seo, Y. K. Kim, B. J. Sung, H. S. Lee, J. I. Lee, S. Y. Park, J. H. Kim, K. Y. Hwang, Y. L. Hyun, Y. H. Jeon, S. Ro, J. M. Cho, T. G. Lee, C. H. Yang, Structural basis for the selective inhibition of JNK1 by the scaffolding protein JIP1 and SP600125. EMBO J 23, 2185-2195 (2004).

19. E. T. Haar, P. Prabakhar, X. Liu, C. Lepre, Crystal structure of the p38 alpha-MAPKAP kinase 2 heterodimer. J Biol Chem 282, 9733-9739 (2007).

20. F. Xin, J. Wu, Crystal structure of the p38 $\alpha$ MAP kinase in complex with a docking peptide from TAB1. Sci China Life Sci 56, 653-660 (2013).

21. T. C. Whisenant, D. T. Ho, R. W. Benz, J. S. Rogers, R. M. Kaake, E. A. Gordon, L. Huang, P. Baldi, L. Bardwell, Computational prediction and experimental verification of new MAP kinase docking sites and substrates including Gli transcription factors. PLoS Comput Biol 6, (2010).

22. H. Saito, F. Posas, Response to hyperosmotic stress. Genetics 192, 289-318 (2012).

23. S. M. Wurgler-Murphy, T. Maeda, E. A. Witten, H. Saito, Regulation of the Saccharomyces cerevisiae HOG1 mitogen-activated protein kinase by the PTP2 and PTP3 protein tyrosine phosphatases. Mol Cell Biol 17, 1289-1297 (1997).

24. S. Grewal, D. M. Molina, L. Bardwell, Mitogen-activated protein kinase (MAPK)docking sites in MAPK kinases function as tethers that are crucial for MAPK regulation 
in vivo. Cell Signal 18, 123-134 (2006).

25. N. Tzarum, N. Komornik, D. Ben Chetrit, D. Engelberg, O. Livnah, DEF pocket in p38alpha facilitates substrate selectivity and mediates autophosphorylation. $J$ Biol Chem 288, 19537-19547 (2013).

26. A. J. Bardwell, E. Frankson, L. Bardwell, Selectivity of docking sites in MAPK kinases. J Biol Chem 284, 13165-13173 (2009).

27. D. T. Ho, A. J. Bardwell, M. Abdollahi, L. Bardwell, A docking site in MKK4 mediates high affinity binding to JNK MAPKs and competes with similar docking sites in JNK substrates. J Biol Chem 278, 32662-32672 (2003).

28. E. Pellegrini, A. Palencia, L. Braun, U. Kapp, A. Bougdour, H. Belrhali, M. W. Bowler, M. A. Hakimi, Structural Basis for the Subversion of MAP Kinase Signaling by an Intrinsically Disordered Parasite Secreted Agonist. Structure 25, 16-26 (2017).

29. L. B. Peterson, M. B. Yaffe, B. Imperiali, Selective mitogen activated protein kinase activity sensors through the application of directionally programmable D domain motifs. Biochemistry 53, 5771-5778 (2014).

30. Y. Tokunaga, K. Takeuchi, H. Takahashi, I. Shimada, Allosteric enhancement of MAP kinase p38 $\alpha$ 's activity and substrate selectivity by docking interactions. Nat Struct Mol Biol 21, 704-711 (2014).

31. T. Ehrenberger, L. C. Cantley, M. B. Yaffe, Computational prediction of protein-protein interactions. Methods Mol Biol 1278, 57-75 (2015).

32. I. Krystkowiak, J. Manguy, N. E. Davey, PSSMSearch: a server for modeling, visualization, proteome-wide discovery and annotation of protein motif specificity determinants. Nucleic Acids Res 46, W235-W241 (2018).

33. A. Subramanian, P. Tamayo, V. K. Mootha, S. Mukherjee, B. L. Ebert, M. A. Gillette, A. Paulovich, S. L. Pomeroy, T. R. Golub, E. S. Lander, J. P. Mesirov, Gene set enrichment analysis: a knowledge-based approach for interpreting genome-wide expression profiles. Proc Natl Acad Sci U S A 102, 15545-15550 (2005).

34. B. Canovas, A. R. Nebreda, Diversity and versatility of p38 kinase signalling in health and disease. Nat Rev Mol Cell Biol 22, 346-366 (2021).

35. S. Anazi, S. Maddirevula, E. Faqeih, H. Alsedairy, F. Alzahrani, H. E. Shamseldin, N. Patel, M. Hashem, N. Ibrahim, F. Abdulwahab, N. Ewida, H. S. Alsaif, H. Al Sharif, W. Alamoudi, A. Kentab, F. A. Bashiri, M. Alnaser, A. H. AlWadei, M. Alfadhel, W. Eyaid, A. Hashem, A. Al Asmari, M. M. Saleh, A. AlSaman, K. A. Alhasan, M. Alsughayir, M. Al Shammari, A. Mahmoud, Z. N. Al-Hassnan, M. Al-Husain, R. Osama Khalil, N. Abd El Meguid, A. Masri, R. Ali, T. Ben-Omran, P. El Fishway, A. Hashish, A. Ercan Sencicek, M. State, A. M. Alazami, M. A. Salih, N. Altassan, S. T. Arold, M. 
Abouelhoda, S. M. Wakil, D. Monies, R. Shaheen, F. S. Alkuraya, Clinical genomics expands the morbid genome of intellectual disability and offers a high diagnostic yield. Mol Psychiatry 22, 615-624 (2017).

36. C. Wentzel, J. E. Sommer, R. Nair, A. Stiefvater, J. B. Sibarita, P. Scheiffele, mSYD1A, a mammalian synapse-defective-1 protein, regulates synaptogenic signaling and vesicle docking. Neuron 78, 1012-1023 (2013).

37. D. A. Fantz, D. Jacobs, D. Glossip, K. Kornfeld, Docking sites on substrate proteins direct extracellular signal-regulated kinase to phosphorylate specific residues. $J$ Biol Chem 276, 27256-27265 (2001).

38. E. P. T. Hertz, T. Kruse, N. E. Davey, B. Lopez-Mendez, J. O. Sigurethsson, G. Montoya, J. V. Olsen, J. Nilsson, A Conserved Motif Provides Binding Specificity to the PP2AB56 Phosphatase. Mol Cell 63, 686-695 (2016).

39. Y. Ueki, T. Kruse, M. B. Weisser, G. N. Sundell, M. S. Y. Larsen, B. L. Mendez, N. P. Jenkins, D. H. Garvanska, L. Cressey, G. Zhang, N. Davey, G. Montoya, Y. Ivarsson, A. N. Kettenbach, J. Nilsson, A Consensus Binding Motif for the PP4 Protein Phosphatase. Mol Cell 76, 953-964 e956 (2019).

40. C. P. Wigington, J. Roy, N. P. Damle, V. K. Yadav, C. Blikstad, E. Resch, C. J. Wong, D. R. Mackay, J. T. Wang, I. Krystkowiak, D. A. Bradburn, E. Tsekitsidou, S. H. Hong, M. A. Kaderali, S. L. Xu, T. Stearns, A. C. Gingras, K. S. Ullman, Y. Ivarsson, N. E. Davey, M. S. Cyert, Systematic Discovery of Short Linear Motifs Decodes Calcineurin Phosphatase Signaling. Mol Cell 79, 342-358 e312 (2020).

41. M. Kataria, S. Mouilleron, M. H. Seo, C. Corbi-Verge, P. M. Kim, F. Uhlmann, A PxL motif promotes timely cell cycle substrate dephosphorylation by the Cdc14 phosphatase. Nat Struct Mol Biol 25, 1093-1102 (2018).

42. I. Faustova, L. Bulatovic, F. Matiyevskaya, E. Valk, M. Örd, M. Loog, A new linear cyclin docking motif that mediates exclusively S-phase CDK-specific signaling. EMBO $J$ 40, e105839 (2021).

43. S. Bandyopadhyay, S. Bhaduri, M. Ord, N. E. Davey, M. Loog, P. M. Pryciak, Comprehensive Analysis of G1 Cyclin Docking Motif Sequences that Control CDK Regulatory Potency In Vivo. Curr Biol 30, 4454-4466 e4455 (2020).

44. G. Gógl, K. D. Schneider, B. J. Yeh, N. Alam, A. N. Nguyen Ba, A. M. Moses, C. Hetényi, A. Reményi, E. L. Weiss, The Structure of an NDR/LATS Kinase-Mob Complex Reveals a Novel Kinase-Coactivator System and Substrate Docking Mechanism. PLoS Biol 13, e1002146 (2015).

45. Y. Ivarsson, R. Arnold, M. McLaughlin, S. Nim, R. Joshi, D. Ray, B. Liu, J. Teyra, T. Pawson, J. Moffat, S. S. Li, S. S. Sidhu, P. M. Kim, Large-scale interaction profiling of PDZ domains through proteomic peptide-phage display using human and viral phage 
peptidomes. Proc Natl Acad Sci U S A 111, 2542-2547 (2014).

46. H. Q. Nguyen, J. Roy, B. Harink, N. P. Damle, N. R. Latorraca, B. C. Baxter, K. Brower, S. A. Longwell, T. Kortemme, K. S. Thorn, M. S. Cyert, P. M. Fordyce, Quantitative mapping of protein-peptide affinity landscapes using spectrally encoded beads. eLife $\mathbf{8}$, (2019).

47. K. Kirsch, A. Zeke, O. Tőke, P. Sok, A. Sethi, A. Sebő, G. S. Kumar, P. Egri, L. Póti Á, P. Gooley, W. Peti, I. Bento, A. Alexa, A. Reményi, Co-regulation of the transcription controlling ATF2 phosphoswitch by JNK and p38. Nat Commun 11, 5769 (2020).

48. D. M. Francis, B. Różycki, D. Koveal, G. Hummer, R. Page, W. Peti, Structural basis of p38 $\alpha$ regulation by hematopoietic tyrosine phosphatase. Nat Chem Biol 7, 916-924 (2011).

49. A. Piserchio, D. M. Francis, D. Koveal, K. N. Dalby, R. Page, W. Peti, R. Ghose, Docking interactions of hematopoietic tyrosine phosphatase with MAP kinases ERK2 and p38a. Biochemistry 51, 8047-8049 (2012).

50. J. Mok, P. M. Kim, H. Y. Lam, S. Piccirillo, X. Zhou, G. R. Jeschke, D. L. Sheridan, S. A. Parker, V. Desai, M. Jwa, E. Cameroni, H. Niu, M. Good, A. Remenyi, J. L. Ma, Y. J. Sheu, H. E. Sassi, R. Sopko, C. S. Chan, C. De Virgilio, N. M. Hollingsworth, W. A. Lim, D. F. Stern, B. Stillman, B. J. Andrews, M. B. Gerstein, M. Snyder, B. E. Turk, Deciphering protein kinase specificity through large-scale analysis of yeast phosphorylation site motifs. Sci Signal 3, ra12 (2010).

51. N. E. Davey, M. H. Seo, V. K. Yadav, J. Jeon, S. Nim, I. Krystkowiak, C. Blikstad, D. Dong, N. Markova, P. M. Kim, Y. Ivarsson, Discovery of short linear motif-mediated interactions through phage display of intrinsically disordered regions of the human proteome. FEBS J 284, 485-498 (2017).

52. A. Zeke, M. Misheva, A. Reményi, M. A. Bogoyevitch, JNK Signaling: Regulation and Functions Based on Complex Protein-Protein Partnerships. Microbiol Mol Biol Rev 80, 793-835 (2016).

53. J. M. Kyriakis, J. Avruch, Mammalian MAPK signal transduction pathways activated by stress and inflammation: a 10-year update. Physiol Rev 92, 689-737 (2012).

54. A. Minden, A. Lin, F. X. Claret, A. Abo, M. Karin, Selective activation of the JNK signaling cascade and c-Jun transcriptional activity by the small GTPases Rac and Cdc42Hs. Cell 81, 1147-1157 (1995).

55. O. A. Coso, M. Chiariello, J. C. Yu, H. Teramoto, P. Crespo, N. Xu, T. Miki, J. S. Gutkind, The small GTP-binding proteins Rac1 and Cdc42 regulate the activity of the JNK/SAPK signaling pathway. Cell 81, 1137-1146 (1995).

56. A. B. Goldberg, E. Cho, C. J. Miller, H. J. Lou, B. E. Turk, Identification of a Substrate- 
selective Exosite within the Metalloproteinase Anthrax Lethal Factor. J Biol Chem 292, 814-825 (2017).

57. D. L. Sheridan, Y. Kong, S. A. Parker, K. N. Dalby, B. E. Turk, Substrate discrimination among mitogen-activated protein kinases through distinct docking sequence motifs. $J$ Biol Chem 283, 19511-19520 (2008).

58. A. F. Thévenin, C. L. Zony, B. J. Bahnson, R. F. Colman, Activation by phosphorylation and purification of human c-Jun N-terminal kinase (JNK) isoforms in milligram amounts. Protein Expr Purif 75, 138-146 (2011).

59. R. D. Gietz, R. H. Schiestl, High-efficiency yeast transformation using the LiAc/SS carrier DNA/PEG method. Nat Protoc 2, 31-34 (2007).

60. C. J. Miller, H. J. Lou, C. Simpson, B. van de Kooij, B. H. Ha, O. S. Fisher, N. L. Pirman, T. J. Boggon, J. Rinehart, M. B. Yaffe, R. Linding, B. E. Turk, Comprehensive profiling of the STE20 kinase family defines features essential for selective substrate targeting and signaling output. PLoS Biol 17, e2006540 (2019).

61. E. Kinoshita-Kikuta, Y. Aoki, E. Kinoshita, T. Koike, Label-free kinase profiling using phosphate affinity polyacrylamide gel electrophoresis. Mol Cell Proteomics 6, 356-366 (2007).

62. J. P. O'Shea, M. F. Chou, S. A. Quader, J. K. Ryan, G. M. Church, D. Schwartz, pLogo: a probabilistic approach to visualizing sequence motifs. Nat Methods 10, 1211-1212 (2013). 